\title{
Theory and application of Fermi pseudo-potential in one dimension
}

\section{Citation}

Wu, Tai Tsun, and Ming Lun Yu. 2002. “Theory and Application of Fermi PseudoPotential in One Dimension." Journal of Mathematical Physics 43 (12): 5949-76. https:// doi.org/10.1063/1.1519940.

\section{Permanent link}

http://nrs.harvard.edu/urn-3:HUL.InstRepos:41555821

\section{Terms of Use}

This article was downloaded from Harvard University's DASH repository, and is made available under the terms and conditions applicable to Other Posted Material, as set forth at http:// nrs.harvard.edu/urn-3:HUL.InstRepos:dash.current.terms-of-use\#LAA

\section{Share Your Story}

The Harvard community has made this article openly available.

Please share how this access benefits you. Submit a story.

Accessibility 


\title{
Theory and application of Fermi pseudo-potential in one dimension
}

\author{
Tai Tsun Wu \\ Gordon McKay Laboratory, Harvard University, \\ Cambridge, Massachusetts, U.S.A., and \\ Theoretical Physics Division, CERN, Geneva, Switzerland \\ and \\ Ming Lun Yu \\ 41019 Pajaro Drive, Fremont, California, U.S.A.円
}

\begin{abstract}
The theory of interaction at one point is developed for the one-dimensional Schrödinger equation. In analog with the three-dimensional case, the resulting interaction is referred to as the Fermi pseudo-potential. The dominant feature of this one-dimensional problem comes from the fact that the real line becomes disconnected when one point is removed. The general interaction at one point is found to be the sum of three terms, the well-known delta-function potential and two Fermi pseudo-potentials, one odd under space reflection and the other even. The odd one gives the proper interpretation for the $\delta^{\prime}(x)$ potential, while the even one is unexpected and more interesting. Among the many applications of these Fermi pseudo-potentials, the simplest one is described. It consists of a superposition of the delta-function potential and the even pseudo-potential applied to two-channel scattering. This simplest application leads to a model of the quantum memory, an essential component of any quantum computer.
\end{abstract}

*Present address: Applied Materials, Inc., 3050 Bowers Ave., Santa Clara, California, U.S.A.

CERN-TH/2002-097

May 2002 


\section{INTRODUCTION}

There are several interrelated motivations for the present investigation. These are discussed in the following.

It was realized several years ago that there are significant differences between scattering in one channel and scattering in two or more coupled channels [1]. For this reason, it may be useful to gain some experience in dealing with coupled channels in general.

The first question is: What is the simplest scattering problem in the case of coupled channels? Once this simplest problem is understood, it is reasonable to expect that many of its features hold also for more general situations. Clearly, for this simplest problem, the number of channels should be chosen to be the smallest, namely two, and the number of spatial dimensions should also be chosen this way, namely one. Thus the scattering problem under consideration, in the time-independent case, deals with the coupled Schrödinger equations

$$
\begin{aligned}
& -\frac{d^{2} \psi_{1}(x)}{d x^{2}}+V_{11}(x) \psi_{1}(x)+V_{12}(x) \psi_{2}(x)=k^{2} \psi_{1}(x), \\
& -\frac{d^{2} \psi_{2}(x)}{d x^{2}}+V_{21}(x) \psi_{1}(x)+V_{22}(x) \psi_{2}(x)=k^{2} \psi_{2}(x),
\end{aligned}
$$

where the $2 \times 2$ matrix potential

$$
V(x)=\left[\begin{array}{ll}
V_{11}(x) & V_{12}(x) \\
V_{21}(x) & V_{22}(x)
\end{array}\right]
$$

is such that it cannot be diagonalized simultaneously for all $x$.

What is the simplest possible choice for this $V(x)$ ? In the case of one channel, the simplest potential is the one that is proportional to the Dirac delta-function $\delta\left(x-x_{0}\right)$. This potential is localized at the one point $x_{0}$, and the corresponding Schrödinger equation is easy to solve. For two coupled channels, it is equally desirable to have the potential localized at one point, say at $x=0$. However, it is not allowed to take $V(x)$ to be the product of $\delta(x)$ and a constant $2 \times 2$ matrix because the diagonalization of this constant matrix decouples the channels.

What is needed is therefore another one-dimensional potential that is localized at one point. With a linear combination of $\delta(x)$ and this new potential, the $2 \times 2$ matrix $V(x)$ can be easily chosen such that it cannot be diagonalized and, hence, the two channels do not decouple.

There are many practical applications of the two-channel scattering problem in one dimension. In this paper, let us restrict ourselves to one such application that is of current interest. The coupled channels can be used as a model for quantum memory; a natural approach to resetting, reading, and writing on a quantum memory is to use scattering from such a quantum memory.

In trying to find this second potential that is localized at one point, it is not necessary to study the coupled Schrödinger equations (1.1); it is sufficient to return to the simpler case of the one-channel Schrödinger equation

$$
\left[-\frac{d^{2}}{d x^{2}}+V(x)\right] \psi(x)=k^{2} \psi(x) .
$$


A natural first guess for this second potential is the derivative of the Dirac deltafunction, i.e., $\delta^{\prime}(x)$. However, the presence of this $\delta^{\prime}(x)$ term in Eq. (1.3) implies that the wave function must be discontinuous at this point $x=0$. But the product of $\delta^{\prime}(x)$ and a function discontinuous at $x=0$ is not well defined. Furthermore, even if this $\delta^{\prime}(x)$ potential is well defined, it is not suitable for the first application to quantum memory. The reason for this will be discussed later in this paper.

A more powerful method is needed to find this desired potential. It is useful here to recall the concept of the Fermi pseudo-potential in three dimensions, which can be written in the form

$$
\delta^{3}(r) \frac{\partial}{\partial r} r
$$

as given by Blatt and Weisskopf [2]. The most far-reaching application of this Fermi pseudo-potential is to the study of many-body systems, as initiated by Huang and Yang [3]. For the ground-state energy per particle of a Bose system of hard spheres, the low-density expansion is known to be

$$
4 \pi a \rho\left[1+\frac{128}{15 \sqrt{\pi}}\left(\rho a^{3}\right)^{1 / 2}+8\left(\frac{4 \pi}{3}-\sqrt{3}\right) \rho a^{3} \ln \left(\rho a^{3}\right)+O\left(\rho a^{3}\right)\right] .
$$

In this expansion, the second term was first obtained by Lee and Yang [4 using the method of binary collision, but the derivation by Lee, Huang and Yang [5] using the Fermi pseudo-potential is somewhat simpler; the third term, which involves the logarithm, was first obtained by using the Fermi pseudo-potential [6]. In the derivation of the third term, it was found inconvenient to use the form (1.4), and thus a limiting process was reintroduced. This point will be of importance in this paper. Thus a great deal is known about the Fermi pseudo-potential in three dimensions.

It is a second motivation for this paper to develop the Fermi pseudo-potential for one-dimensional scattering. In many cases, once a theory has been developed for three dimensions, it is straightforward to repeat the development for one dimension. In the present case of the Fermi pseudo-potential, this is not the case. Furthermore, the result for one dimension seems qualitatively different from that for three dimensions.

For clarity of presentation, this paper is organized into two parts: Part A for the theory of the Fermi pseudo-potential in one dimension, and Part B for its application to quantum computing. Needless to say, these two parts are closely related to each other. The sections are numbered consecutively throughout the paper.

\section{Part A}

\section{Theory of One-Dimensional Fermi Pseudo-Potential}

\section{INTERACTION AT ONE POINT}

In the absence of $V$, the Hamiltonian of Eq. (1.3) is

$$
H_{0}=-\frac{d^{2}}{d x^{2}}
$$


for real $x$, where the right-hand side is suitably interpreted so that it is self-adjoint. Let $k$ be purely imaginary; define a real, positive $\kappa$ by

$$
\kappa=-i k \text {. }
$$

For such a $k$, the Green's function, or resolvent, for this $H_{0}$ satisfies the differential equation

$$
\left(-\frac{d^{2}}{d x^{2}}+\kappa^{2}\right) R_{\kappa}^{(0)}\left(x, x^{\prime}\right)=\delta\left(x-x^{\prime}\right)
$$

and is given explicitly by

$$
R_{\kappa}^{(0)}\left(x, x^{\prime}\right)=(2 \kappa)^{-1} e^{-\kappa\left|x-x^{\prime}\right|}
$$

Let a potential $V$ be added to this $H_{0}$ to give

$$
H=H_{0}+V
$$

which is also self-adjoint. Again for $\kappa$ positive, the Green's function, or resolvent, for this $H$ satisfies, similar to Eq. (2.3),

$$
\left(H+\kappa^{2}\right) R_{\kappa}\left(x, x^{\prime}\right)=\delta\left(x-x^{\prime}\right) .
$$

The interaction $V$ is said to be at the one point $x_{0}$ if Eq. (2.6) implies that Eq. (2.3), with $R_{\kappa}^{(0)}\left(x, x^{\prime}\right)$ replaced by $R_{\kappa}\left(x, x^{\prime}\right)$, is satisfied for all $x$ except $x=x_{0}$.

Because of translational symmetry, this $x_{0}$ is chosen to be 0 throughout this paper. It is a consequence of Eq. (2.4) and the symmetry of the Green's function that this definition of an interaction at one point implies

$$
R_{\kappa}\left(x, x^{\prime}\right)=\frac{1}{2 \kappa}\left[e^{-\kappa\left|x-x^{\prime}\right|}-f\left(\kappa ; \operatorname{sg} x, \operatorname{sg} x^{\prime}\right) e^{-\kappa\left(|x|+\left|x^{\prime}\right|\right)}\right]
$$

where $\operatorname{sg} x$ and $\operatorname{sg} x^{\prime}$ mean the sign of $x$ and the sign of $x^{\prime}$, respectively.

Since Eq. (2.7) is the starting point for the present paper, this is the appropriate place to add the following comments.

(1) In the present case of one dimension, the real line with the point $x=0$ removed is not connected. This is a qualitative difference between one dimension and higher dimensions.

(2) It is because of this property that the $f$ in Eq. (2.7) can depend on the signs of $x$ and $x^{\prime}$.

(3) In the three-dimensional case, the Fermi pseudo-potential can be obtained in the following way: Take the self-adjoint Hamiltonian $-\nabla^{2}$, where $\nabla^{2}$ is the threedimensional Laplacian, and restrict it to functions that are zero at $\mathbf{r}=0$; the selfadjoint extensions [7] of this restricted operator give the Fermi pseudo-potential, i.e., these self-adjoint extensions can be written as the sum of $-\nabla^{2}$ and (1.4) multiplied by a constant. Such a procedure applied to the case of one dimension does not give Eq. (2.7). 
It is useful to write out explicitly the $f$ of Eq. (2.7) as:

$$
f\left(\kappa ; \operatorname{sg} x, \operatorname{sg} x^{\prime}\right)= \begin{cases}f_{1}(\kappa), & \text { for } x>0, x^{\prime}>0 \\ f_{2}(\kappa), & \text { for } x<0, x^{\prime}>0 \\ f_{3}(\kappa), & \text { for } x<0, x^{\prime}<0 \\ f_{4}(\kappa), & \text { for } x>0, x^{\prime}<0\end{cases}
$$

following the four quadrants in the $x-x^{\prime}$ plane. Note that all of these $f$ 's are dimensionless.

\section{RESOLVENT EQUATION}

In view of Eq. (2.7), it is most convenient to study the resolvent equation in coordinate representation:

$$
R_{\kappa_{1}}\left(x, x^{\prime}\right)-R_{\kappa_{2}}\left(x, x^{\prime}\right)+\left(\kappa_{1}^{2}-\kappa_{2}^{2}\right) \int_{-\infty}^{\infty} d x^{\prime \prime} R_{\kappa_{1}}\left(x, x^{\prime \prime}\right) R_{\kappa_{2}}\left(x^{\prime \prime}, x^{\prime}\right)=0,
$$

where $\kappa_{1}$ and $\kappa_{2}$ are two values of $\kappa$.

The substitution of Eq. (2.7) into this resolvent equation (3.1) gives, after a lengthy calculation,

$$
\begin{aligned}
& \frac{1}{\kappa_{1}-\kappa_{2}} f\left(\kappa_{1} ; \operatorname{sg} x, \operatorname{sg} x^{\prime}\right)+\frac{1}{\kappa_{1}+\kappa_{2}} f\left(\kappa_{1} ; \operatorname{sg} x,-\operatorname{sg} x^{\prime}\right) \\
& -\frac{1}{\kappa_{1}-\kappa_{2}} f\left(\kappa_{2} ; \operatorname{sg} x, \operatorname{sg} x^{\prime}\right)+\frac{1}{\kappa_{1}+\kappa_{2}} f\left(\kappa_{2} ;-\operatorname{sg} x, \operatorname{sg} x^{\prime}\right) \\
& -\frac{1}{\kappa_{1}+\kappa_{2}}\left[f\left(\kappa_{1} ; \operatorname{sg} x,-\right) f\left(\kappa_{2} ;-, \operatorname{sg} x^{\prime}\right)+f\left(\kappa_{1} ; \operatorname{sg} x,+\right) f\left(\kappa_{2} ;+, \operatorname{sg} x^{\prime}\right)\right] \\
& =0
\end{aligned}
$$

for $\kappa_{1} \neq \kappa_{2}$. This is the resolvent equation for the interaction at the point $x=0$ as defined in Sec. [II].

Equation (3.2) has the following symmetry properties besides space reflection.

(1) Since $f\left(\kappa ; \operatorname{sg} x, \operatorname{sg} x^{\prime}\right)$ is dimensionless, there is no scale for $\kappa$. Thus, Eq. (3.2) is invariant under the scale change

$$
\kappa_{1} \rightarrow \lambda \kappa_{1} \quad \text { and } \quad \kappa_{2} \rightarrow \lambda \kappa_{2}
$$

Note that $\lambda$ is positive since the $\kappa$ 's are positive.

(2) There is an additional symmetry

$$
f\left(\kappa ; \operatorname{sg} x, \operatorname{sg} x^{\prime}\right) \rightarrow-\operatorname{sg} x \operatorname{sg} x^{\prime} f\left(\frac{1}{\kappa} ; \operatorname{sg} x, \operatorname{sg} x^{\prime}\right) .
$$

This discrete symmetry is going to play an important role in this paper. In terms of the $f_{j}(\kappa)$ defined in Eq. (2.8), this symmetry is

$$
\kappa \rightarrow \frac{1}{\kappa}
$$




$$
\begin{array}{ll}
f_{1} \rightarrow-f_{1}, & f_{3} \rightarrow-f_{3}, \\
f_{2} \rightarrow f_{2}, & f_{4} \rightarrow f_{4} .
\end{array}
$$

The next task is to solve the resolvent equation (3.2) for $f\left(\kappa ; \operatorname{sg} x, \operatorname{sg} x^{\prime}\right)$. Since differential equations are easier to deal with than difference equations, it is convenient to take the limit $\kappa_{1} \rightarrow \kappa_{2}$. In this limit, Eq. (3.2) reduces to

$$
\begin{aligned}
& \frac{d}{d \kappa} f\left(\kappa ; \operatorname{sg} x, \operatorname{sg} x^{\prime}\right)+\frac{1}{2 \kappa}\left[f\left(\kappa ; \operatorname{sg} x,-\operatorname{sg} x^{\prime}\right)+f\left(\kappa ;-\operatorname{sg} x, \operatorname{sg} x^{\prime}\right)\right] \\
& -\frac{1}{2 \kappa}\left[f(\kappa ; \operatorname{sg} x,-) f\left(\kappa ;-, \operatorname{sg} x^{\prime}\right)+f(\kappa ; \operatorname{sg} x,+) f\left(\kappa ;+, \operatorname{sg} x^{\prime}\right)\right] \\
& \quad=0
\end{aligned}
$$

In terms of the $f_{j}(\kappa)$ of Eq. (2.8), this differential equation (3.6) consists of the following four equations by taking various signs for $x$ and $x^{\prime}$ :

$$
\begin{array}{r}
f_{1}^{\prime}(\kappa)+\frac{1}{2 \kappa}\left[f_{2}(\kappa)+f_{4}(\kappa)\right]-\frac{1}{2 \kappa}\left[f_{2}(\kappa) f_{4}(\kappa)+f_{1}(\kappa)^{2}\right]=0, \\
f_{2}^{\prime}(\kappa)+\frac{1}{2 \kappa}\left[f_{1}(\kappa)+f_{3}(\kappa)\right]-\frac{1}{2 \kappa}\left[f_{2}(\kappa) f_{3}(\kappa)+f_{1}(\kappa) f_{2}(\kappa)\right]=0, \\
f_{3}^{\prime}(\kappa)+\frac{1}{2 \kappa}\left[f_{2}(\kappa)+f_{4}(\kappa)\right]-\frac{1}{2 \kappa}\left[f_{3}(\kappa)^{2}+f_{2}(\kappa) f_{4}(\kappa)\right]=0, \\
f_{4}^{\prime}(\kappa)+\frac{1}{2 \kappa}\left[f_{1}(\kappa)+f_{3}(\kappa)\right]-\frac{1}{2 \kappa}\left[f_{3}(\kappa) f_{4}(\kappa)+f_{1}(\kappa) f_{4}(\kappa)\right]=0 .
\end{array}
$$

An examination of these four differential equations shows the important role played by the combination $f_{1}(\kappa)+f_{3}(\kappa)$, which appears twice in Eq. (3.7b) and twice in Eq. (3.7d). Define $F(\kappa)$ up to an additive constant by

$$
F^{\prime}(\kappa)=\frac{1}{2 \kappa}\left[f_{1}(\kappa)+f_{3}(\kappa)\right]
$$

In terms of this $F(\kappa)$, Eqs. (3.7b) and (3.7d) take the form

$$
f_{2}^{\prime}(\kappa)+F^{\prime}(\kappa)-f_{2}(\kappa) F^{\prime}(\kappa)=0 ; \quad f_{4}^{\prime}(\kappa)+F^{\prime}(\kappa)-f_{4}(\kappa) F^{\prime}(\kappa)=0 .
$$

Integration of Eqs. (3.9) gives $f_{2}(\kappa)$ and $f_{4}(\kappa)$ in terms of $F(\kappa)$ :

$$
f_{2}(\kappa)=1+c_{2} e^{F(\kappa)} ; \quad f_{4}(\kappa)=1+c_{4} e^{F(\kappa)},
$$

where $c_{2}$ and $c_{4}$ are two arbitrary constants of integration. Similarly, subtracting Eq. (3.7c) from Eq. (3.7a) gives

$$
\frac{d}{d \kappa}\left[f_{1}(\kappa)-f_{3}(\kappa)\right]=\frac{1}{2 \kappa}\left[f_{1}(\kappa)^{2}-f_{3}(\kappa)^{2}\right]=F^{\prime}(\kappa)\left[f_{1}(\kappa)-f_{3}(\kappa)\right],
$$

or

$$
f_{1}(\kappa)-f_{3}(\kappa)=2 c_{3} e^{F(\kappa)}
$$


where $c_{3}$ is another arbitrary constant of integration.

It remains to determine $F(\kappa)$, which satisfies the second-order ordinary differential equation obtained from adding Eqs. (3.7a) and (3.7g):

$$
2 \kappa \frac{d}{d \kappa} \kappa \frac{d}{d \kappa} F(\kappa)=\left[\kappa \frac{d}{d \kappa} F(\kappa)\right]^{2}-1+\left(c_{3}^{2}+c_{2} c_{4}\right) e^{2 F(\kappa)} .
$$

The solution of this equation is straightforward but somewhat lengthy and is thus relegated to Appendix A.

The results are as follows:

$$
\begin{aligned}
& f_{1}(\kappa)=\frac{-\sqrt{c_{3}^{2}+c_{2} c_{4}-c_{1}^{2}}\left[c_{0} \kappa+\left(c_{0} \kappa\right)^{-1}\right]-2 c_{3}}{\sqrt{c_{3}^{2}+c_{2} c_{4}-c_{1}^{2}}\left[c_{0} \kappa-\left(c_{0} \kappa\right)^{-1}\right]+2 c_{1}}, \\
& f_{2}(\kappa)=1-\frac{2 c_{2}}{\sqrt{c_{3}^{2}+c_{2} c_{4}-c_{1}^{2}}\left[c_{0} \kappa-\left(c_{0} \kappa\right)^{-1}\right]+2 c_{1}}, \\
& f_{3}(\kappa)=\frac{-\sqrt{c_{3}^{2}+c_{2} c_{4}-c_{1}^{2}}\left[c_{0} \kappa+\left(c_{0} \kappa\right)^{-1}\right]+2 c_{3}}{\sqrt{c_{3}^{2}+c_{2} c_{4}-c_{1}^{2}}\left[c_{0} \kappa-\left(c_{0} \kappa\right)^{-1}\right]+2 c_{1}}, \\
& f_{4}(\kappa)=1-\frac{2 c_{4}}{\sqrt{c_{3}^{2}+c_{2} c_{4}-c_{1}^{2}}\left[c_{0} \kappa-\left(c_{0} \kappa\right)^{-1}\right]+2 c_{1}},
\end{aligned}
$$

when

$$
c_{3}^{2}+c_{2} c_{4}-c_{1}^{2}>0
$$

Similarly,

$$
\begin{aligned}
& f_{1}(\kappa)=\frac{-\sqrt{c_{1}^{2}-c_{3}^{2}-c_{2} c_{4}}\left[c_{0} \kappa-\left(c_{0} \kappa\right)^{-1}\right]-2 c_{3}}{\sqrt{c_{1}^{2}-c_{3}^{2}-c_{2} c_{4}}\left[c_{0} \kappa-\left(c_{0} \kappa\right)^{-1}\right]+2 c_{1}}, \\
& f_{2}(\kappa)=1-\frac{2 c_{2}}{\sqrt{c_{1}^{2}-c_{3}^{2}-c_{2} c_{4}}\left[c_{0} \kappa-\left(c_{0} \kappa\right)^{-1}\right]+2 c_{1}}, \\
& f_{3}(\kappa)=\frac{-\sqrt{c_{1}^{2}-c_{3}^{2}-c_{2} c_{4}}\left[c_{0} \kappa-\left(c_{0} \kappa\right)^{-1}\right]+2 c_{3}}{\sqrt{c_{1}^{2}-c_{3}^{2}-c_{2} c_{4}}\left[c_{0} \kappa-\left(c_{0} \kappa\right)^{-1}\right]+2 c_{1}}, \\
& f_{4}(\kappa)=1-\frac{2 c_{4}}{\sqrt{c_{1}^{2}-c_{3}^{2}-c_{2} c_{4}}\left[c_{0} \kappa-\left(c_{0} \kappa\right)^{-1}\right]+2 c_{1}},
\end{aligned}
$$

when

$$
c_{3}^{2}+c_{2} c_{4}-c_{1}^{2}<0
$$

In Eqs. (3.14) and (3.16), $c_{0}>0$ and all square roots are also positive. Although there are five constants $c_{0}, c_{1}, c_{2}, c_{3}$ and $c_{4}$, effectively there are four because all the quantities do not change under

$$
c_{j} \rightarrow \lambda c_{j}
$$


for $j=1,2,3,4$, and $\lambda>0$.

It remains to discuss briefly the case

$$
c_{3}^{2}+c_{2} c_{4}-c_{1}^{2}=0 .
$$

This case, which was in fact the first case worked out and also the most important one as discussed in Sec. IV, can be recovered by taking the limit $c_{3}^{2}+c_{2} c_{4}-c_{1}^{2} \rightarrow 0$ together with either $c_{0} \rightarrow 0$ or $c_{0} \rightarrow \infty$. These two limiting cases are to be considered separately. For definiteness, they are applied to Eqs. (3.16).

(1) $c_{3}^{2}+c_{2} c_{4}-c_{1}^{2} \rightarrow 0$ and $c_{0} \rightarrow 0$, such that

$$
\gamma=c_{0}^{-1} \sqrt{c_{1}^{2}-c_{3}^{2}-c_{2} c_{4}}
$$

is fixed; $\gamma>0$. In this limit,

$$
\begin{aligned}
& f_{1}(\kappa)=\frac{\gamma-2 c_{3} \kappa}{\gamma+2 c_{1} \kappa}, \\
& f_{2}(\kappa)=1-\frac{2 c_{2} \kappa}{\gamma+2 c_{1} \kappa}, \\
& f_{3}(\kappa)=\frac{\gamma+2 c_{3} \kappa}{\gamma+2 c_{1} \kappa}, \\
& f_{4}(\kappa)=1-\frac{2 c_{4} \kappa}{\gamma+2 c_{1} \kappa} .
\end{aligned}
$$

(2) $c_{3}^{2}+c_{2} c_{4}-c_{1}^{2} \rightarrow 0$ and $c_{0} \rightarrow \infty$, such that

$$
\gamma=c_{0} \sqrt{c_{1}^{2}-c_{3}^{2}-c_{2} c_{4}}
$$

is fixed; $\gamma>0$. In this limit,

$$
\begin{aligned}
& f_{1}(\kappa)=\frac{-\gamma \kappa-2 c_{3}}{\gamma \kappa+2 c_{1}}, \\
& f_{2}(\kappa)=1-\frac{2 c_{2}}{\gamma \kappa+2 c_{1}}, \\
& f_{3}(\kappa)=\frac{-\gamma \kappa+2 c_{3}}{\gamma \kappa+2 c_{1}}, \\
& f_{4}(\kappa)=1-\frac{2 c_{4}}{\gamma \kappa+2 c_{1}} .
\end{aligned}
$$

Note that Eqs. (3.21) and (3.23) are related by the discrete symmetry (3.5) provided that the sign of $c_{3}$ is reversed. The same results also follow from Eqs. (3.14).

\section{INTERACTION POTENTIALS}

Naively, one would expect it to be straightforward to determine the potential when the Green's function (resolvent) is known. It does not turn out to be so straightforward, and this section is devoted to solving this problem. 
The substitution of Eqs. (2.1) and (2.5) into Eq. (2.6) gives

$$
V(x) R_{\kappa}\left(x, x^{\prime}\right)=\delta\left(x-x^{\prime}\right)-\left(-\frac{d^{2}}{d x^{2}}+\kappa^{2}\right) R_{\kappa}\left(x, x^{\prime}\right),
$$

or

$$
V(x) R_{\kappa}\left(x, x^{\prime}\right)=-\left(-\frac{d^{2}}{d x^{2}}+\kappa^{2}\right)\left[R_{\kappa}\left(x, x^{\prime}\right)-R_{\kappa}^{(0)}\left(x, x^{\prime}\right)\right]
$$

with the last term defined by Eq. (2.3) or Eq. (2.4). This should determine $V(x)$; that this $V(x)$ does not depend on $\kappa$ is a consequence of $R_{\kappa}\left(x, x^{\prime}\right)$ satisfying the resolvent equation.

More generally, the left-hand side of Eq. (4.2) may be an integral, and this equation takes the form

$$
\int_{-\infty}^{\infty} d x^{\prime \prime} V\left(x, x^{\prime \prime}\right) R_{\kappa}\left(x^{\prime \prime}, x^{\prime}\right)=-\left(-\frac{d^{2}}{d x^{2}}+\kappa^{2}\right)\left[R_{\kappa}\left(x, x^{\prime}\right)-R_{\kappa}^{(0)}\left(x, x^{\prime}\right)\right] .
$$

If $V(x)$ exists, then

$$
V\left(x, x^{\prime}\right)=V(x) \delta\left(x-x^{\prime}\right)
$$

Since Eq. (4.2) is simpler than Eq. (4.3), it is useful to study Eq. (4.2) first even though it is less general. The substitution of Eq. (2.7) into Eq. (4.2) gives

$$
V(x) R_{\kappa}\left(x, x^{\prime}\right)=\frac{1}{2 \kappa}\left(-\frac{d^{2}}{d x^{2}}+\kappa^{2}\right) f\left(\kappa ; \operatorname{sg} x, \operatorname{sg} x^{\prime}\right) e^{-\kappa\left(|x|+\left|x^{\prime}\right|\right)} .
$$

The difficulty is to give a proper interpretation to this equation. The right-hand side contains a term

$$
f\left(\kappa ; \operatorname{sg} x, \operatorname{sg} x^{\prime}\right) \delta(x) e^{-\kappa\left|x^{\prime}\right|},
$$

obtained by applying the differential operator to the exponential. As seen from Eqs. (3.14) for example, this expression (4.6) is in general the product of $\delta(x)$ and a function discontinuous at $x=0$. The only reasonable interpretation of such a product is

$$
\alpha(x) \delta(x)=\frac{1}{2}\left[\left(\lim _{x \rightarrow 0+}+\lim _{x \rightarrow 0-}\right) \alpha(x)\right] \delta(x) .
$$

As mentioned above, there are effectively four parameters in the solutions as given by either Eqs. (3.14) or Eqs. (3.16). Since the symmetry of the Green's function implies that $c_{2}=c_{4}$, the number of parameters is reduced by 1 . Therefore, the potential $V\left(x, x^{\prime}\right)$ depends on three parameters. That there are three parameters instead of two is a major surprise, and this fact is to play a central role in Part B of this paper, where this interaction potential is applied to study certain aspects of quantum computing.

The three pieces of $V\left(x, x^{\prime}\right)$ are of different levels of complication. They are studied in the following three subsections. 


\section{A. $\delta(x)$ potential}

The simplest piece is the well-known $\delta(x)$ potential, where

$$
V_{1}(x)=g_{1} \delta(x)
$$

and

$$
V_{1}\left(x, x^{\prime}\right)=g_{1} \delta(x) \delta\left(x-x^{\prime}\right)
$$

or equivalently

$$
V_{1}\left(x, x^{\prime}\right)=g_{1} \delta(x) \delta\left(x^{\prime}\right)
$$

For this potential, the differential equation (2.6) is well defined. Its solution is

$$
R_{\kappa}\left(x, x^{\prime}\right)=\frac{1}{2 \kappa}\left[e^{-\kappa\left|x-x^{\prime}\right|}-\frac{g_{1}}{2 \kappa+g_{1}} e^{-\kappa\left(|x|+\left|x^{\prime}\right|\right)}\right] .
$$

Comparison with Eq. (2.7) gives

$$
f\left(\kappa ; \operatorname{sg} x, \operatorname{sg} x^{\prime}\right)=\frac{g_{1}}{2 \kappa+g_{1}}
$$

independent of the signs of $x$ and $x^{\prime}$. By Eq. (2.8), this is

$$
f_{1}(\kappa)=f_{2}(\kappa)=f_{3}(\kappa)=f_{4}(\kappa)=\frac{g_{1}}{2 \kappa+g_{1}} .
$$

This is a special case of Eqs. (3.21) with

$$
c_{1}=c_{2}=c_{4}=\frac{\gamma}{g_{1}} \quad \text { and } \quad c_{3}=0
$$

It is instructive to recover Eq. (4.8) from Eq. (4.12) by using Eq. (4.5). Since $f\left(\kappa ; \operatorname{sg} x, \operatorname{sg} x^{\prime}\right)$ does not depend on the signs of $x$ and $x^{\prime}$, Eq. (4.5) gives

$$
\begin{aligned}
V(x) & =\left[\frac{g_{1}}{2 \kappa+g_{1}} \delta(x) e^{-\kappa\left|x^{\prime}\right|}\right] /\left\{\frac{1}{2 \kappa}\left[e^{-\kappa\left|x-x^{\prime}\right|}-\frac{g_{1}}{2 \kappa+g_{1}} e^{-\kappa\left(|x|+\left|x^{\prime}\right|\right)}\right]\right\} \\
& =g_{1} \delta(x) .
\end{aligned}
$$

This $V(x)$ is even in $x$.

\section{B. $\delta^{\prime}(x)$ potential}

As already discussed in Sec. I, the potential $\delta^{\prime}(x)$ is not acceptable because, in the Schrödinger equation, there is a product of $\delta^{\prime}(x)$ and a function discontinuous at $x=0$. While $\delta(x)$ is a potential, $\delta^{\prime}(x)$ has to be understood as a Fermi pseudopotential in much the same way as the expression (1.4) in three dimensions. 
Since $\delta^{\prime}(x)$ is odd in $x$, the resolvent must satisfy

$$
f_{1}(\kappa)=-f_{3}(\kappa) \quad \text { and } \quad f_{2}(\kappa)=f_{4}(\kappa) .
$$

Next, consider the formal Eq. (2.6) with this $\delta^{\prime}(x)$ potential

$$
\left[-\frac{d^{2}}{d x^{2}}+g_{2} \delta^{\prime}(x)+\kappa^{2}\right] R_{\kappa}\left(x, x^{\prime}\right)=\delta\left(x-x^{\prime}\right) .
$$

Since every term on the left-hand side is of dimension $x^{-2}$ times that of $R_{\kappa}\left(x, x^{\prime}\right)$, the resolvent $R_{\kappa}\left(x, x^{\prime}\right)$ must be of the form

$$
\kappa^{-1} \text { function of } \kappa x \text { and } \kappa x^{\prime}
$$

A comparison with Eq. (2.7) then shows that $f\left(\kappa ; \operatorname{sg} x, \operatorname{sg} x^{\prime}\right)$ is independent of $\kappa$. This is satisfied with

$$
f_{1}(\kappa)=\alpha, \quad f_{3}(\kappa)=-\alpha, \quad \text { and } \quad f_{2}(\kappa)=f_{4}(\kappa)=-\beta,
$$

with

$$
2 \beta+\alpha^{2}+\beta^{2}=0
$$

With the resolvent known, it is now possible to define the Fermi pseudo-potential

$$
V_{2}(x)=g_{2} \delta_{p}^{\prime}(x)
$$

Omitting the argument $\kappa$ in $f$, Eq. (4.5) takes the form

$$
g_{2} \delta_{p}^{\prime}(x) R_{\kappa}\left(x, x^{\prime}\right)=\frac{1}{2 \kappa}\left(-\frac{d^{2}}{d x^{2}}+\kappa^{2}\right) f\left(\operatorname{sg} x, \operatorname{sg} x^{\prime}\right) e^{-\kappa\left(|x|+\left|x^{\prime}\right|\right)} .
$$

Let $x^{\prime}$ be positive. Then

$$
\left(-\frac{d^{2}}{d x^{2}}+\kappa^{2}\right) f\left(\operatorname{sg} x, \operatorname{sg} x^{\prime}\right) e^{-\kappa|x|}=-\left(f_{1}-f_{2}\right) \delta^{\prime}(x)+\kappa\left(f_{1}+f_{2}\right) \delta(x) .
$$

This expression has not only a $\delta^{\prime}(x)$ term, but also a $\delta(x)$ term. For the left-hand side of Eq. (4.21), it is necessary to evaluate, using Eq. (4.7),

$$
\begin{aligned}
\left.R_{\kappa}\left(x, x^{\prime}\right)\right|_{x=0} & =\left[e^{-\kappa\left(x^{\prime}-x\right)}-f(\operatorname{sg} x,+) e^{-\kappa\left(|x|+x^{\prime}\right)}\right]_{x=0} \\
& =e^{-\kappa x^{\prime}}\left[1-\frac{1}{2}\left(\lim _{x \rightarrow 0+}+\lim _{x \rightarrow 0-}\right) f(\operatorname{sg} x,+) e^{-\kappa|x|}\right] \\
& =e^{-\kappa x^{\prime}}\left[1-\frac{1}{2}\left(f_{1}+f_{2}\right)\right]
\end{aligned}
$$


and

$$
\begin{aligned}
\frac{d}{d x} & {\left[e^{-\kappa\left(x^{\prime}-x\right)}-f(\operatorname{sg} x,+) e^{-\kappa\left(|x|+x^{\prime}\right)}\right]_{x=0} } \\
& =\kappa\left[e^{-\kappa\left(x^{\prime}-x\right)}+(\operatorname{sg} x) f(\operatorname{sg} x,+) e^{-\kappa\left(|x|+x^{\prime}\right)}\right]_{x=0} \\
& =\kappa e^{-\kappa x^{\prime}}\left[1+\frac{1}{2}\left(\lim _{x \rightarrow 0+}+\lim _{x \rightarrow 0-}\right)(\operatorname{sg} x) f(\operatorname{sg} x,+) e^{-\kappa|x|}\right] \\
& =\kappa e^{-\kappa x^{\prime}}\left[1+\frac{1}{2}\left(f_{1}-f_{2}\right)\right] .
\end{aligned}
$$

Suppose the $\delta_{p}^{\prime}(x)$ on the left-hand side of Eq. (4.21) is replaced by $\delta^{\prime}(x)$. Then a comparison of Eq. (4.22) with Eqs. (4.23) and (4.24) gives

$$
g_{2}\left[1-\frac{1}{2}\left(f_{1}+f_{2}\right)\right]=f_{1}-f_{2} ; \quad g_{2}\left[1+\frac{1}{2}\left(f_{1}-f_{2}\right)\right]=f_{1}+f_{2},
$$

where the identity $x \delta^{\prime}(x)=-\delta(x)$ has been used. These are the conditions for $x^{\prime}>0$; similar conditions for $x^{\prime}<0$ are

$$
g_{2}\left[1-\frac{1}{2}\left(f_{4}+f_{3}\right)\right]=f_{4}-f_{3} ; \quad g_{2}\left[-1+\frac{1}{2}\left(f_{4}-f_{3}\right)\right]=f_{4}+f_{3} .
$$

Solving Eqs. (4.25) and (4.26) gives

$$
f_{1}=\frac{g_{2}}{1+g_{2}^{2} / 4} ; \quad f_{3}=\frac{-g_{2}}{1+g_{2}^{2} / 4} ; \quad f_{2}=f_{4}=\frac{g_{2}^{2} / 2}{1+g_{2}^{2} / 4} .
$$

This is consistent with the previous Eqs. (4.18) and (4.19).

Where is the difficulty explained in Sec. I? Another way of asking the same question is: How does $\delta_{p}^{\prime}(x)$ differ from $\delta^{\prime}(x)$ ?

The answer is to be found in the first step of Eq. (4.24). In differentiating the quantity on the left-hand side of Eq. (4.24), the factor $f(\operatorname{sg} x,+)$ is not differentiated. In other words, the term with $(d / d x) f(\operatorname{sg} x,+)$ has been omitted; if this term were not omitted, there would be a $\delta(x)$, precisely the difficulty explained in Sec. I.

The situation is therefore entirely similar to the Fermi pseudo-potential in three dimensions, where the operator (1.4) performs the function of removing a term proportional to $1 / r$. Here, what $\delta_{p}^{\prime}(x)$ does is

$$
\delta_{p}^{\prime}(x) g(x)=\delta^{\prime}(x) \tilde{g}(x),
$$

where

$$
\tilde{g}(x)= \begin{cases}g(x)-\lim _{x \rightarrow 0+} g(x), & \text { for } x>0 \\ g(x)-\lim _{x \rightarrow 0-} g(x), & \text { for } x<0\end{cases}
$$

This removes the discontinuity of $g(x)$ at $x=0$, which is precisely what is needed. 


\section{Third potential}

At the beginning of this investigation it was thought that, in one dimension, there was one potential (subsection [VA) and one pseudo-potential (subsection [VB). But the detailed analysis of the resolvent equation in Sec. III shows that there are three independent parameters in the resolvent, and hence there is an independent third potential, or a second pseudo-potential in one dimension.

This third potential is most easily understood through the discrete symmetry (3.4). Let $c=2 / g_{1}$. Then, from Eq. (4.13), the resolvent for the $\delta(x)$ potential is given by

$$
f_{1}(\kappa)=f_{2}(\kappa)=f_{3}(\kappa)=f_{4}(\kappa)=\frac{1}{1+c \kappa} .
$$

Application of the discrete symmetry (3.4) to Eq. (4.30) gives the result that the resolvent for the third potential is expressed by

$$
f_{1}(\kappa)=f_{3}(\kappa)=\frac{-\kappa}{\kappa+c} ; \quad f_{2}(\kappa)=f_{4}(\kappa)=\frac{\kappa}{\kappa+c} .
$$

In particular, similar to the resolvent of the first pseudo-potential as given by Eq. (4.27), this resolvent is also not continuous. The relation between the resolvents as expressed by Eqs. (4.30) and (4.31) is a special case of that between Eqs. (3.21) and (3.23).

It remains to determine the potential, or more precisely the pseudo-potential, from Eq. (4.31), which can be written more succinctly as

$$
f\left(\kappa ; \operatorname{sg} x, \operatorname{sg} x^{\prime}\right)=-\frac{\kappa}{\kappa+c} \operatorname{sg} x \operatorname{sg} x^{\prime} .
$$

Therefore, for the present case of the third potential $V_{3}\left(x, x^{\prime}\right)$, Eq. (4.3) takes the form

$$
\begin{gathered}
\int_{-\infty}^{\infty} d x^{\prime \prime} V_{3}\left(x, x^{\prime \prime}\right)\left[e^{-\kappa\left|x^{\prime \prime}-x^{\prime}\right|}+\frac{\kappa}{\kappa+c} \operatorname{sg} x^{\prime \prime} \operatorname{sg} x^{\prime} e^{-\kappa\left(\left|x^{\prime \prime}\right|+\left|x^{\prime}\right|\right)}\right] \\
=\frac{\kappa}{\kappa+c}\left(-\frac{d^{2}}{d x^{2}}+\kappa^{2}\right)\left[\operatorname{sg} x \operatorname{sg} x^{\prime} e^{-\kappa\left(|x|+\left|x^{\prime}\right|\right)}\right] .
\end{gathered}
$$

Differentiation of the right-hand side gives

$$
\frac{d}{d x}\left[\operatorname{sg} x e^{-\kappa|x|}\right]=2 \delta(x)-\kappa e^{-\kappa|x|},
$$

and hence

$$
\left(-\frac{d^{2}}{d x^{2}}+\kappa^{2}\right)\left[\operatorname{sg} x e^{-\kappa|x|}\right]=-2 \delta^{\prime}(x) .
$$

Thus Eq. (4.33) for $V_{3}\left(x, x^{\prime}\right)$ is explicitly

$$
\begin{aligned}
\int_{-\infty}^{\infty} & d x^{\prime \prime} V_{3}\left(x, x^{\prime \prime}\right)\left[e^{-\kappa\left|x^{\prime \prime}-x^{\prime}\right|}+\frac{\kappa}{\kappa+c} \operatorname{sg} x^{\prime \prime} \operatorname{sg} x^{\prime} e^{-\kappa\left(\left|x^{\prime \prime}\right|+\left|x^{\prime}\right|\right)}\right] \\
= & -\frac{2 \kappa}{\kappa+c} \delta^{\prime}(x) \operatorname{sg} x^{\prime} e^{-\kappa\left|x^{\prime}\right|} .
\end{aligned}
$$


The task is to make sense of this equation and to determine $V_{3}\left(x, x^{\prime}\right)$.

That the resolvent equation is satisfied means that Eq. (4.35), properly understood, does lead to a $V_{3}\left(x, x^{\prime}\right)$. By Eqs. (4.28) and (4.29), the $\delta^{\prime}(x)$ on the right-hand side of Eq. (4.35) can be replaced by $\delta_{p}^{\prime}(x)$, because it is not multiplied by a discontinuous function of $x$. Therefore, $V_{3}\left(x, x^{\prime}\right)$ is expected to be proportional to $\delta_{p}^{\prime}(x)$; that $\delta_{p}^{\prime}(x)$ is used instead of $\delta^{\prime}(x)$ is due to the development in subsection IVB. With these considerations, an examination of Eq. (4.35) indicates that

$$
V_{3}\left(x, x^{\prime}\right)=g_{3} \delta_{p}^{\prime}(x) \delta_{p}^{\prime}\left(x^{\prime}\right)
$$

See also Eq. (4.20).

It remains to substitute Eq. (4.36) into Eq. (4.35) to find the relation between the two constants $g_{3}$ and $c$ :

$$
\begin{aligned}
& g_{3} \int_{-\infty}^{\infty} d x^{\prime \prime} \delta_{p}^{\prime}\left(x^{\prime \prime}\right)\left[e^{-\kappa\left|x^{\prime \prime}-x^{\prime}\right|}+\frac{\kappa}{\kappa+c} \operatorname{sg} x^{\prime \prime} \operatorname{sg} x^{\prime} e^{-\kappa\left(\left|x^{\prime \prime}\right|+\left|x^{\prime}\right|\right)}\right] \\
& =-\frac{2 \kappa}{\kappa+c} \operatorname{sg} x^{\prime} e^{-\kappa\left|x^{\prime}\right|}
\end{aligned}
$$

The evaluation of the first integral is straightforward because $e^{-\kappa\left|x^{\prime \prime}-x^{\prime}\right|}$ is continuous:

$$
\begin{aligned}
\int_{-\infty}^{\infty} d x^{\prime \prime} \delta_{p}^{\prime}\left(x^{\prime \prime}\right) e^{-\kappa\left|x^{\prime \prime}-x^{\prime}\right|} & =\int_{-\infty}^{\infty} d x^{\prime \prime} \delta^{\prime}\left(x^{\prime \prime}\right) e^{-\kappa\left|x^{\prime \prime}-x^{\prime}\right|} \\
& =-\kappa \operatorname{sg} x^{\prime} e^{-\kappa\left|x^{\prime}\right|}
\end{aligned}
$$

After the removal of the common factor

$$
\kappa \operatorname{sg} x^{\prime} e^{-\kappa\left|x^{\prime}\right|},
$$

Eq. (4.37) reduces to

$$
g_{3}\left[-1+\frac{1}{\kappa+c} \int_{-\infty}^{\infty} d x^{\prime \prime} \delta_{p}^{\prime}\left(x^{\prime \prime}\right) \operatorname{sg} x^{\prime \prime} e^{-\kappa\left|x^{\prime \prime}\right|}\right]=-\frac{2}{\kappa+c} .
$$

This integral can be evaluated using Eqs. (4.28) and (4.29):

$$
\int_{-\infty}^{\infty} d x^{\prime \prime} \delta_{p}^{\prime}\left(x^{\prime \prime}\right) \operatorname{sg} x^{\prime \prime} e^{-\kappa\left|x^{\prime \prime}\right|}=-\int_{-\infty}^{\infty} d x^{\prime \prime} \delta\left(x^{\prime \prime}\right) \operatorname{sg} x^{\prime \prime} \frac{d}{d x^{\prime \prime}} e^{-\kappa\left|x^{\prime \prime}\right|}=\kappa .
$$

Therefore, Eq. (4.39) is simply

$$
g_{3}\left[-1+\frac{\kappa}{\kappa+c}\right]=-\frac{2}{\kappa+c} \quad \text { or } \quad g_{3}=\frac{2}{c} .
$$

This is the desired relation.

It is merely a matter of terminology whether this pseudo-potential $V_{3}\left(x, x^{\prime}\right)$ as given by Eq. (4.36) is called a local potential or not. 
In summary, the three potentials $V_{1}, V_{2}$, and $V_{3}$ are given by Eqs. (4.8), (4.20), and (4.36). Thus the most general Fermi pseudo-potential for the interaction at one point in one dimension is

$$
\begin{aligned}
V\left(x, x^{\prime}\right) & =V_{1}\left(x, x^{\prime}\right)+V_{2}\left(x, x^{\prime}\right)+V_{3}\left(x, x^{\prime}\right) \\
& =g_{1} \delta(x) \delta\left(x-x^{\prime}\right)+g_{2} \delta_{p}^{\prime}(x) \delta\left(x-x^{\prime}\right)+g_{3} \delta_{p}^{\prime}(x) \delta_{p}^{\prime}\left(x^{\prime}\right) .
\end{aligned}
$$

From the above experience of working with $\delta_{p}^{\prime}(x)$ and the fact that the product of $\delta^{\prime}(x)$ and a function discontinuous at $x=0$ is not meaningful, from here on the convention will be adopted that $\delta^{\prime}(x)$ always means $\delta_{p}^{\prime}(x)$. With this convention, Eq. (4.42) is written as

$$
V\left(x, x^{\prime}\right)=g_{1} \delta(x) \delta\left(x-x^{\prime}\right)+g_{2} \delta^{\prime}(x) \delta\left(x-x^{\prime}\right)+g_{3} \delta^{\prime}(x) \delta^{\prime}\left(x^{\prime}\right) .
$$

Equation (4.43) can be rewritten in a prettier form as follows. Since

$$
\delta(x) \delta\left(x-x^{\prime}\right)=\delta(x) \delta\left(x^{\prime}\right)
$$

and

$$
\begin{aligned}
\delta^{\prime}(x) \delta\left(x-x^{\prime}\right) & =\delta^{\prime}(x) \delta\left(x^{\prime}-x\right) \\
& =\delta^{\prime}(x)\left[\delta\left(x^{\prime}\right)-x \delta^{\prime}\left(x^{\prime}\right)\right] \\
& =\delta^{\prime}(x) \delta\left(x^{\prime}\right)+\delta(x) \delta^{\prime}\left(x^{\prime}\right),
\end{aligned}
$$

where use has been made of the identity $\delta^{\prime}(x) x=-\delta(x)$, a general Fermi pseudopotential (4.43) can be written as

$$
V\left(x, x^{\prime}\right)=g_{1} \delta(x) \delta\left(x^{\prime}\right)+g_{2}\left[\delta^{\prime}(x) \delta\left(x^{\prime}\right)+\delta(x) \delta^{\prime}\left(x^{\prime}\right)\right]+g_{3} \delta^{\prime}(x) \delta^{\prime}\left(x^{\prime}\right) .
$$

As already mentioned, the first and last terms are even while the middle term is odd. That is, under space inversion

$$
x \rightarrow-x \text { and } \quad x^{\prime} \rightarrow-x^{\prime},
$$

the coupling constants transform as

$$
g_{1} \rightarrow g_{1} ; \quad g_{2} \rightarrow-g_{2} ; \quad g_{3} \rightarrow g_{3} .
$$

\section{SOLVING THE SCHRÖDINGER EQUATION}

In applying the Fermi pseudo-potential to various problems, such as the one to be treated in Part B of this paper, the resolvent equation is difficult to use and it is much more convenient to employ the prescription of Sec. [V to the Schrödinger equation.

This section is devoted to studying the equation

$$
\left(-\frac{d^{2}}{d x^{2}}+\kappa^{2}\right) R_{\kappa}\left(x, x^{\prime}\right)+\int_{-\infty}^{\infty} d x^{\prime \prime} V\left(x^{\prime}, x^{\prime \prime}\right) R_{\kappa}\left(x^{\prime \prime}, x^{\prime}\right)=\delta\left(x-x^{\prime}\right),
$$


where $V\left(x, x^{\prime}\right)$ is the Fermi pseudo-potential as given by Eq. (4.42). On the one hand, this is an equation for this $V\left(x, x^{\prime}\right)$. On the other hand, the procedure of this section is directly applicable to the Schrödinger equation, which differs from Eq. (5.1) only in the absence of the $\delta\left(x-x^{\prime}\right)$ term on the right-hand side.

This section serves two distinct purposes. First, the parameters in the known resolvent of Sec. [1], especially Eqs. (3.14) and (3.16), are to be related to the coupling constants $g_{1}, g_{2}$, and $g_{3}$ in Eq. (4.42). This will give an explicit verification of consistency of the prescriptions given in Sec. IV. Secondly, the procedure to be followed here serves as a useful introduction to the slightly more complicated problem of the next section, where a two-channel scattering by a Fermi pseudo-potential is taken to be a model for a quantum memory.

The solution $R_{\kappa}\left(x, x^{\prime}\right)$ is given, as in the general case, by Eq. (2.7). The substitution of Eq. (2.7) into Eq. (5.1) gives

$$
\left(-\frac{d^{2}}{d x^{2}}+\kappa^{2}\right)\left[-\frac{1}{2 \kappa} f\left(\kappa ; \operatorname{sg} x, \operatorname{sg} x^{\prime}\right) e^{-\kappa\left(|x|+\left|x^{\prime}\right|\right)}\right]+\int_{-\infty}^{\infty} d x^{\prime \prime} V\left(x, x^{\prime \prime}\right) R_{\kappa}\left(x^{\prime \prime}, x^{\prime}\right)=0 .
$$

Since the first term has been evaluated by Eq. (4.22), Eq. (5.2) can be written alternatively as

$$
\begin{aligned}
2 \kappa & \int_{-\infty}^{\infty} d x^{\prime \prime} V\left(x, x^{\prime \prime}\right) R_{\kappa}\left(x^{\prime \prime}, x^{\prime}\right) \\
& =e^{-\kappa\left|x^{\prime}\right|} \begin{cases}\left\{-\left[f_{1}(\kappa)-f_{2}(\kappa)\right] \delta^{\prime}(x)+\kappa\left[f_{1}(\kappa)+f_{2}(\kappa)\right] \delta(x)\right\}, & \text { for } x^{\prime}>0 \\
\left\{-\left[f_{4}(\kappa)-f_{3}(\kappa)\right] \delta^{\prime}(x)+\kappa\left[f_{4}(\kappa)+f_{3}(\kappa)\right] \delta(x)\right\}, & \text { for } x^{\prime}<0 .\end{cases}
\end{aligned}
$$

Using the knowledge gained from Sec. IV, a fairly lengthy calculation gives more explicitly

$$
\begin{gathered}
{\left[g_{1} \delta(x)+g_{2} \delta^{\prime}(x)\right] e^{-\kappa\left|x^{\prime}\right|} \begin{cases}\left\{1-\frac{1}{2}\left[f_{1}(\kappa)+f_{2}(\kappa)\right]\right\}, & \text { for } x^{\prime}>0 \\
\left\{1-\frac{1}{2}\left[f_{4}(\kappa)+f_{3}(\kappa)\right]\right\}, & \text { for } x^{\prime}<0\end{cases} } \\
+\left[g_{2} \delta(x)+g_{3} \delta^{\prime}(x)\right] \kappa e^{-\kappa\left|x^{\prime}\right|} \begin{cases}\left\{-1+\frac{1}{2}\left[-f_{1}(\kappa)+f_{2}(\kappa)\right]\right\}, & \text { for } x^{\prime}>0 \\
\left\{1+\frac{1}{2}\left[-f_{4}(\kappa)+f_{3}(\kappa)\right]\right\}, & \text { for } x^{\prime}<0\end{cases} \\
=e^{-\kappa\left|x^{\prime}\right|} \begin{cases}\left\{-\left[f_{1}(\kappa)-f_{2}(\kappa)\right] \delta^{\prime}(x)+\kappa\left[f_{1}(\kappa)+f_{2}(\kappa)\right] \delta(x)\right\}, & \text { for } x^{\prime}>0 \\
\left\{-\left[f_{4}(\kappa)-f_{3}(\kappa)\right] \delta^{\prime}(x)+\kappa\left[f_{4}(\kappa)+f_{3}(\kappa)\right] \delta(x)\right\}, & \text { for } x^{\prime}<0,\end{cases}
\end{gathered}
$$

where Eq. (4.45) has been used. In Eq. (5.4), all dependences on $x^{\prime}$ cancel out. It 
therefore only remains to identify the coefficients of $\delta(x)$ and $\delta^{\prime}(x)$. The results are

$$
\begin{aligned}
& g_{1}\{\left.1-\frac{1}{2}\left[f_{1}(\kappa)+f_{2}(\kappa)\right]\right\}+\kappa g_{2}\left\{-1+\frac{1}{2}\left[-f_{1}(\kappa)+f_{2}(\kappa)\right]\right\} \\
&=\kappa\left[f_{1}(\kappa)+f_{2}(\kappa)\right], \\
& g_{2}\{1\left.-\frac{1}{2}\left[f_{1}(\kappa)+f_{2}(\kappa)\right]\right\}+\kappa g_{3}\left\{-1+\frac{1}{2}\left[-f_{1}(\kappa)+f_{2}(\kappa)\right]\right\} \\
&=-\left[f_{1}(\kappa)-f_{2}(\kappa)\right], \\
& g_{1}\left\{1-\frac{1}{2}\left[f_{4}(\kappa)+f_{3}(\kappa)\right]\right\}+\kappa g_{2}\left\{1+\frac{1}{2}\left[-f_{4}(\kappa)+f_{3}(\kappa)\right]\right\} \\
& \quad=\kappa\left[f_{4}(\kappa)+f_{3}(\kappa)\right], \\
& g_{2}\left\{1-\frac{1}{2}\left[f_{4}(\kappa)+f_{3}(\kappa)\right]\right\}+\kappa g_{3}\left\{1+\frac{1}{2}\left[-f_{4}(\kappa)+f_{3}(\kappa)\right]\right\} \\
& \quad=-\left[f_{4}(\kappa)-f_{3}(\kappa)\right] .
\end{aligned}
$$

Solving Eqs. (5.5) gives

$$
\begin{aligned}
& f_{1}(\kappa)=D^{-1}\left[-g_{3} \kappa+2 g_{2}-g_{1} \kappa^{-1}\right], \\
& f_{3}(\kappa)=D^{-1}\left[-g_{3} \kappa-2 g_{2}-g_{1} \kappa^{-1}\right], \\
& f_{2}(\kappa)=f_{4}(\kappa)=1+D^{-1} \frac{1}{2}\left(4+g_{1} g_{3}-g_{2}^{2}\right),
\end{aligned}
$$

where

$$
D=g_{3} \kappa-\frac{1}{2}\left(4-g_{1} g_{3}+g_{2}^{2}\right)-g_{1} \kappa^{-1} .
$$

Equations (5.6) are to be compared with Eqs. (3.14) and (3.16). First, this gives a deeper understanding why there are the two distinct cases (3.14) and (3.16). Equations (3.14) correspond to the situation where $g_{1}$ and $g_{3}$ have the same sign, while Eqs. (3.16) correspond to $g_{1}$ and $g_{3}$ having opposite signs. Secondly, in both cases, it is seen immediately from Eqs. (5.6) that $c_{2}=c_{4}$, a fact that has been used before. The results of expressing the five $c$ 's in terms of the three $g$ 's are the following:

$$
\begin{aligned}
c_{0} & =\sqrt{\left|g_{3} / g_{1}\right|}, \\
c_{1} & =\frac{1}{4}\left(4-g_{1} g_{3}+g_{2}^{2}\right), \\
c_{2} & =c_{4}=\frac{1}{4}\left(4+g_{1} g_{3}-g_{2}^{2}\right), \\
c_{3} & =g_{2} .
\end{aligned}
$$

Here use has been made of the scale invariance (3.18). [Strictly speaking, the righthand sides of the three Eqs. (5.9a-c) should all be multiplied by the factor $\operatorname{sg} g_{3}$. This factor has been omitted because it has no consequences.]

For completeness and also for later use, let the scattering matrix be written down. This involves returning to the more familiar variable $k$ through Eq. (2.2) and then 
letting $x^{\prime} \rightarrow \pm \infty$. After analytic continuation to positive values of $k$, the $S$-matrix is a $2 \times 2$ matrix

$$
S=\left[\begin{array}{ll}
S_{++} & S_{+-} \\
S_{-+} & S_{--}
\end{array}\right]
$$

where + denotes propagation in the $+x$ direction, and - in the $-x$ direction. For any interaction at the point $x=0$, it follows from Eqs. (2.7) and (2.8) that

$$
\begin{aligned}
S & =\left[\begin{array}{cc}
1-f(-i k ;+,-) & -f(-i k ;-,-) \\
-f(-i k ;+,+) & 1-f(-i k ;-,+)
\end{array}\right] \\
& =\left[\begin{array}{cc}
1-f_{4}(-i k) & -f_{3}(-i k) \\
-f_{1}(-i k) & 1-f_{2}(-i k)
\end{array}\right] .
\end{aligned}
$$

Equations (5.6) and (5.7) then give explicitly

$$
\begin{aligned}
S= & {\left[i g_{3} k+\frac{1}{2}\left(4-g_{1} g_{3}+g_{2}^{2}\right)+i g_{1} k^{-1}\right]^{-1} } \\
& \times\left[\begin{array}{cc}
\frac{1}{2}\left(4+g_{1} g_{3}-g_{2}^{2}\right) & i g_{3} k-2 g_{2}-i g_{1} k^{-1} \\
i g_{3} k+2 g_{2}-i g_{1} k^{-1} & \frac{1}{2}\left(4+g_{1} g_{3}-g_{2}^{2}\right)
\end{array}\right],
\end{aligned}
$$

which is unitary.

An interesting special case is that with $g_{2}=0$; in this case, the pseudo-potential is even and there is left-right symmetry. Explicitly, in this case $g_{2}=0$, the $S$-matrix is

$$
S=\left[i g_{3} k+\frac{1}{2}\left(4-g_{1} g_{3}\right)+i g_{1} k^{-1}\right]^{-1}\left[\begin{array}{cc}
\frac{1}{2}\left(4+g_{1} g_{3}\right) & i g_{3} k-i g_{1} k^{-1} \\
i g_{3} k-i g_{1} k^{-1} & \frac{1}{2}\left(4+g_{1} g_{3}\right)
\end{array}\right] .
$$

This special case $g_{2}=0$, generalized to the case of coupled channels, is going to play a central role in Part B of this paper.

This completes the present discussion of the theory of Fermi pseudo-potential in one dimension. Attention is now turned to the first application of this theory.

\section{Part B}

\section{Application of One-Dimensional Fermi Pseudo-Potential}

\section{MODEL FOR QUANTUM MEMORY}

There are many possible applications of the Fermi pseudo-potential in one dimension. As an example, it is intriguing to ask under what conditions, if any, Bethe's hypothesis [8, 9] still holds when the delta-function potential is replaced by the $V\left(x, x^{\prime}\right)$ of Eq. (4.42). As a first application, however, it is more desirable to begin with a case where the Fermi pseudo-potential is used in a relatively simple situation of current interest. 
For decades, computer components have become smaller and smaller, and this trend is expected to continue [10]. When some of the components become sufficiently small, as to be expected in the not-too-distant future, they need to be described in general by quantum mechanics. No matter how quantum computing is to develop in the future, one important component is necessarily the quantum memory, sometimes called the quantum register. The main function of any quantum memory is to store a quantum state.

In order for a quantum memory to be useful, it must be possible to alter the quantum state in the memory in a controlled way. This can only be accomplished by sending a signal from outside of the memory. In other words, the quantum state in the memory is to be controlled by a scattering process [11].

It is the purpose of Sec. V1 to propose a simple model for quantum memory. First, in order to have scattering processes, at least one space dimension is necessary. Otherwise there is no possibility of interference between the incident wave and the scattered wave. As perhaps to be expected, this interference is of central importance. Since the state in the memory must include at least two independent quantum states, it is simplest to describe the quantum memory using the coupled Schrödinger equations for two channels. This is essentially Eq. (1.1) in the Introduction.

It remains to make the simplest choice for the $2 \times 2$ matrix potential $V(x)$ of Eq. (1.2). This simplest choice, the Fermi pseudo-potential in one dimension, has been investigated systematically in Part $\mathrm{A}$ of this paper, the general result being given by Eq. (4.42).

The symmetry properties of this $V\left(x, x^{\prime}\right)$ under space inversion have been discussed at the end of Sec. IV. In particular, it is symmetrical if $g_{2}=0$. In order for the model to be suitable for quantum memory, it is essential to concentrate on this special case. The reason is that, only in this case, do the even wave $\cos k x$ and the odd wave $\sin k x$ not mix. This is also the basis for the comment in the Introduction, after Eq. (1.3), why the $\delta^{\prime}(x)$ potential is not suitable for quantum memory. That this absence of mixing is important is discussed further in Sec. VII for a more general setting.

With this understanding and choice, the present model for the quantum memory is described by the one-dimensional coupled Schrödinger equations

$$
\begin{aligned}
& -\frac{d^{2} \psi_{1}(x)}{d x^{2}}+\int_{-\infty}^{\infty} d x^{\prime}\left[V_{11}\left(x, x^{\prime}\right) \psi_{1}\left(x^{\prime}\right)+V_{12}\left(x, x^{\prime}\right) \psi_{2}\left(x^{\prime}\right)\right]=k^{2} \psi_{1}(x), \\
& -\frac{d^{2} \psi_{2}(x)}{d x^{2}}+\int_{-\infty}^{\infty} d x^{\prime}\left[V_{21}\left(x, x^{\prime}\right) \psi_{1}\left(x^{\prime}\right)+V_{22}\left(x, x^{\prime}\right) \psi_{2}\left(x^{\prime}\right)\right]=k^{2} \psi_{2}(x),
\end{aligned}
$$

with the $2 \times 2$ matrix potential

$$
\begin{aligned}
V\left(x, x^{\prime}\right) & =\left[\begin{array}{ll}
V_{11}\left(x, x^{\prime}\right) & V_{12}\left(x, x^{\prime}\right) \\
V_{21}\left(x, x^{\prime}\right) & V_{22}\left(x, x^{\prime}\right)
\end{array}\right] \\
& =\left[\begin{array}{cc}
g_{3} \delta^{\prime}(x) \delta^{\prime}\left(x^{\prime}\right) & g_{1} \delta(x) \delta\left(x^{\prime}\right) \\
g_{1} \delta(x) \delta\left(x^{\prime}\right) & -g_{3} \delta^{\prime}(x) \delta^{\prime}\left(x^{\prime}\right)
\end{array}\right] .
\end{aligned}
$$


A more elegant way to write this potential is

$$
V\left(x, x^{\prime}\right)=g_{1} \delta(x) \delta\left(x^{\prime}\right) \sigma_{1}+g_{3} \delta^{\prime}(x) \delta^{\prime}\left(x^{\prime}\right) \sigma_{3},
$$

where the $\sigma$ 's are the Pauli matrices.

When $g_{2}=0$, the potential $g_{1} \delta(x) \delta\left(x^{\prime}\right)$ does not act on the odd wave, and similarly the potential $g_{3} \delta^{\prime}(x) \delta^{\prime}\left(x^{\prime}\right)$ does not act on the even wave. The first part of this claim is easy to obtain, and the second part follows from the definition (4.28) of $\delta_{p}^{\prime}(x)$. Alternatively, they can be seen from Eq. (5.13), where $S_{++}=S_{--}$and $S_{+-}=S_{-+}$. For the even wave, the scattering phase shift is given by

$$
S_{++}+S_{+-}=\frac{2 k-i g_{1}}{2 k+i g_{1}}
$$

independent of $g_{3}$, while, for the odd wave, it is

$$
S_{++}-S_{+-}=\frac{2-i g_{3} k}{2+i g_{3} k}
$$

independent of $g_{1}$. Therefore, for the present case of two coupled channels as described by Eqs. (6.1) and (6.2), the $S$-matrix for the even and odd cases can be expressed in terms of these quantities as follows. Consider first the case for the odd wave; since the $g_{1}$ term does not contribute and can be neglected, the $V\left(x, x^{\prime}\right)$ of Eq. (6.2) effectively reduces to

$$
V\left(x, x^{\prime}\right)=\left[\begin{array}{cc}
g_{3} \delta^{\prime}(x) \delta^{\prime}\left(x^{\prime}\right) & 0 \\
0 & -g_{3} \delta^{\prime}(x) \delta^{\prime}\left(x^{\prime}\right)
\end{array}\right]
$$

which is diagonal, meaning that $\psi_{1}(x)$ and $\psi_{2}(x)$ do not couple. Since the behaviors of the two channels differ only in the sign of $g_{3}$, the $S$-matrix for this odd case is given by Eq. (6.5), or more explicitly

$$
S_{-}(k)=\left[\begin{array}{cc}
\frac{2-i g_{3} k}{2+i g_{3} k} & 0 \\
0 & \frac{2+i g_{3} k}{2-i g_{3} k}
\end{array}\right] \text {. }
$$

It is instructive to rewrite this expression in terms of $\sigma_{3}$ :

$$
S_{-}(k)=\frac{\left(4-g_{3}^{2} k^{2}\right)-4 i g_{3} k \sigma_{3}}{4+g_{3}^{2} k^{2}}=\exp \left[-i \sigma_{3}\left(2 \tan ^{-1} \frac{g_{3} k}{2}\right)\right] .
$$

For the even wave, it is merely necessary to replace the right-hand side of Eq. (6.5) by that of Eq. (6.4), and also $\sigma_{3}$ by $\sigma_{1}$. Therefore Eq. (6.8) leads to

$$
S_{+}(k)=\frac{\left(4 k^{2}-g_{1}^{2}\right)-4 i g_{1} k \sigma_{1}}{4 k^{2}+g_{1}^{2}}=\exp \left[-i \sigma_{1}\left(2 \tan ^{-1} \frac{g_{1}}{2 k}\right)\right]
$$


or

$$
S_{+}(k)=\left[\begin{array}{cc}
\frac{4 k^{2}-g_{1}^{2}}{4 k^{2}+g_{1}^{2}} & \frac{-4 i g_{1} k}{4 k^{2}+g_{1}^{2}} \\
\frac{-4 i g_{1} k}{4 k^{2}+g_{1}^{2}} & \frac{4 k^{2}-g_{1}^{2}}{4 k^{2}+g_{1}^{2}}
\end{array}\right] .
$$

When neither $g_{1}$ nor $g_{3}$ is zero, any given element $S$ of $\mathrm{SU}(2)$ can be expressed as a finite product of $S_{+}(k)$ and $S_{-}(k)$, i.e.,

$$
S=S\left(k_{1}\right) S\left(k_{2}\right) \cdots S\left(k_{m}\right),
$$

where each $S\left(k_{i}\right)$ is suitably chosen as $S_{+}\left(k_{i}\right)$ or $S_{-}\left(k_{i}\right)$.

In the language of scattering theory, the meaning of $S_{+}(k)$ is as follows. [The meaning of $S_{-}(k)$ is similar.] The "in" field is

$$
\Psi^{\text {in }}=\left[\begin{array}{c}
a_{1}^{\text {in }} \\
a_{2}^{\text {in }}
\end{array}\right] e^{-i k|x|},
$$

while the "out" field is

$$
\Psi^{\text {out }}=\left[\begin{array}{l}
a_{1}^{\text {out }} \\
a_{2}^{\text {out }}
\end{array}\right] e^{i k|x|} .
$$

Then

$$
\left[\begin{array}{l}
a_{1}^{\text {out }} \\
a_{2}^{\text {out }}
\end{array}\right]=S_{+}(k)\left[\begin{array}{l}
a_{1}^{\text {in }} \\
a_{2}^{\text {in }}
\end{array}\right]
$$

In other words, the quantum state in the memory is $\left[\begin{array}{c}a_{1}^{\text {in }} \\ a_{2}^{\text {in }}\end{array}\right]$ before scattering, and $\left[\begin{array}{l}a_{1}^{\text {out }} \\ a_{2}^{\text {out }}\end{array}\right]$ after scattering. These states before and after scattering are related by Eq. (6.14).

For any memory, classical or quantum, the basic operations are write, read, and reset. Of these three operations, writing is the simplest: Given the initial state $\left[\begin{array}{l}a_{1}^{\text {in }} \\ a_{2}^{\text {in }}\end{array}\right]$ and the desired final state $\left[\begin{array}{l}a_{1}^{\text {out }} \\ a_{2}^{\text {out }}\end{array}\right]$, there is a desired $S$-matrix $S$. Express this particular $S$ by Eq. (6.11) as a finite product; then the writing is accomplished by a sequence of these $m$ scatterings.

The question may be asked: While the final state $\left[\begin{array}{l}a_{1}^{\text {out }} \\ a_{2}^{\text {out }}\end{array}\right]$ is the desired state to be stored in the quantum memory, how can one know what the initial state $\left[\begin{array}{l}a_{1}^{\text {in }} \\ a_{2}^{\text {in }}\end{array}\right]$ is? This is where the idea of a standard state $s$ is needed. "Resetting" means changing the content of the quantum memory, whatever it is, to the standard state $s$. For writing, the initial state is the standard state, i.e.,

$$
\left[\begin{array}{l}
a_{1}^{\text {in }} \\
a_{2}^{\text {in }}
\end{array}\right]=s .
$$


In other words, before writing on a quantum memory, it is first reset so that Eq. (6.15) is satisfied. The standard state can be chosen to be any quantum state; however, once chosen, the choice is rarely altered.

Since scattering from a quantum memory leads to a unitary transformation of the quantum state in the memory, resetting cannot be accomplished without first finding out the content of the quantum memory. In other words, the first step of "resetting" is "reading." After the content of the quantum memory is known, say $\left[\begin{array}{l}a_{1}^{\text {in }} \\ a_{2}^{\text {in }}\end{array}\right]$, "resetting" involves finding a sequence of scattering $S\left(k_{j}\right), j=1,2, \ldots \quad n$, via Eq. (6.11) such that the resulting $S$ has the property

$$
S\left[\begin{array}{l}
a_{1}^{\text {in }} \\
a_{2}^{\text {in }}
\end{array}\right]=s .
$$

In summary, if "reading" can be accomplished, then so can "resetting"; if "resetting" can be accomplished, so can "writing."

The main task here is therefore to discuss, within the present model, the operation of reading a quantum memory. More precisely, what is involved is the following. When the quantum state in a memory, $\left[\begin{array}{l}a_{1}^{\text {in }} \\ a_{2}^{\text {in }}\end{array}\right]$, is not known, find a suitably chosen sequence of incident waves $\cos k_{i} x$ or $\sin k_{i} x$ such that the knowledge about the field can be used to determine the values of $a_{1}^{\text {in }}$ and $a_{2}^{\text {in }}$. After this determination, the quantum memory is returned to the initial state $\left[\begin{array}{c}a_{1}^{\text {in }} \\ a_{2}^{\text {in }}\end{array}\right]$. [The last step is similar to the classical case in which a core memory is read from an initial state and then returned to it.]

Let the quantum state in the memory be $\left[\begin{array}{l}a_{1} \\ a_{2}\end{array}\right]$; the problem is to determine the values of $a_{1}$ and $a_{2}$ by scattering from this state. Suppose an odd wave is used for the first scattering; then the two-component wave function for $x>0$ is given explicitly by

$$
\psi(x)=\left[\begin{array}{l}
\psi_{1}(x) \\
\psi_{2}(x)
\end{array}\right]=\left[\begin{array}{l}
a_{1} \\
a_{2}
\end{array}\right] e^{-i k x}+\left[\begin{array}{c}
a_{1} e^{i \phi_{-}} \\
a_{2} e^{-i \phi_{-}}
\end{array}\right] e^{i k x},
$$

where, by Eq. (6.8),

$$
\phi_{-}=-2 \tan ^{-1} \frac{1}{2} g_{3} k
$$

In particular,

$$
\psi(x)^{\dagger} \psi(x)=2\left[1+\cos 2 k x \cos \phi_{-}-\left(\left|a_{1}\right|^{2}-\left|a_{2}\right|^{2}\right) \sin 2 k x \sin \phi_{-}\right]
$$

because $\left|a_{1}\right|^{2}+\left|a_{2}\right|^{2}=1$. Therefore, this scattering process gives, through the interference term, the quantity

$$
A_{1}=\left|a_{1}\right|^{2}-\left|a_{2}\right|^{2} .
$$


Similarly, if the quantum state in the memory is first returned to the original state by a suitable scattering, then a second scattering with an even wave gives, again through the interference term, the second quantity

$$
A_{2}=\frac{1}{2}\left[\left|a_{1}+a_{2}\right|^{2}+\left|a_{1}-a_{2}\right|^{2}\right]=2 \operatorname{Re} a_{1}^{*} a_{2}
$$

These two quantities, $A_{1}$ and $A_{2}$, are sufficient to determine the values of the complex numbers $a_{1}$ and $a_{2}$, except for a common phase. In order to determine this common phase, it is simplest to use the known standard state $s$. For example, a further interference with this standard state using, say, the odd wave gives a third quantity

$$
A_{3}=\left|a_{1}+s_{1}\right|^{2}-\left|a_{2}+s_{2}\right|^{2} .
$$

Since $s_{1}$ and $s_{2}$ are known, these three quantities $A_{1}, A_{2}$ and $A_{3}$ determine $a_{1}$ and $a_{2}$. Returning once more to the original quantum state presents no problem.

This completes the description of the present model of the quantum memory, including the operations of writing, reading, and resetting.

The advantages of this model, based on the Fermi pseudo-potential in one dimension, are its simplicity and its being completely explicit. On the one hand, such an explicit model plays an essential role in the initial understanding of some aspects of a new problem. On the other hand, the usefulness of such a model really lies in the possibility of opening a line of inquiry into these aspects. This is to be discussed in some detail in the next section. That is, in Sec. VII an attempt is to be made to present a general picture concerning the quantum memory, emphasizing the operations of writing, reading, and resetting, all accomplished by repeated scattering.

Some simplifying assumptions introduced in the model of this section are clearly not needed in the general setting of the next section. An example is the choice of using the Fermi pseudo-potential $V\left(x, x^{\prime}\right)$ of Eq. (6.2); another one is the use of the Schrödinger equation (6.1) in one dimension. Thus the generalization to the Schrödinger equation in three dimensions with a more general potential is immediate but the results are less explicit. The further generalization to renormalized quantum field theory also does not present any obstacle.

What is less clear, and most important, is the role played by the condition $g_{2}=$ 0 , used throughout this section. This condition is closely related to, and makes it possible to use, the even waves and the odd waves. In order to appreciate this point, take instead the incoming wave as, say, from the direction of the $-x$ axis, i.e.,

$$
\Psi^{\text {in }}=\left[\begin{array}{l}
a_{1}^{\text {in }} \\
a_{2}^{\text {in }}
\end{array}\right] e^{-i k x} .
$$

This is a superposition of an even wave and an odd wave. Since the Schrödinger equation is linear, the even part is operated on by the $S_{+}(k)$ of Eq. (6.10), and the odd part by the $S_{-}(k)$ of Eq. (6.7). Since $g_{1}$ and $g_{3}$ are not zero and thus these $S_{+}(k)$ and $S_{-}(k)$ are not equal, the quantum state in the memory for an outgoing wave in the $+x$ direction is different from that for an outgoing wave in the $-x$ direction. In other words, in order to determine the quantum state in the memory after scattering with the $\Psi^{\text {in }}$ of Eq. (6.23), it is necessary to detect the direction of the outgoing wave. 
In order for a quantum memory to behave as a memory, i.e., as the storage for a quantum state, it is essential that what is in the memory does not depend on the behavior of the scattered wave. Indeed, from the point of view of scattering theory, this characterizes quantum memories. Therefore, for the present model with the Fermi pseudo-potential, some incident waves, such as the even wave and the odd wave, are acceptable or "admissible," while many others, such as $e^{-i k x}$ of Eq. (6.23), are not "admissible."

This concept of admissible incident waves is central, not only for the present model but also in general. This is the first topic to be discussed in the next section.

\section{GENERALIZATION}

It is the purpose of this section to give a general description of quantum memories. This is to be accomplished by extracting the dominant features from the model of Sec. VI on the basis of the Fermi pseudo-potential in one dimension.

In order to extract the dominant features, consider first the following two generalizations, the first one obvious and the second one less so.

First, that the potential is the Fermi pseudo-potential is not necessary. In other words, the matrix potential $V\left(x, x^{\prime}\right)$ of Eq. (6.2) can take a fairly general form. That $g_{2}$ is zero translates into the condition that this $V\left(x, x^{\prime}\right)$ is symmetrical, i.e.,

$$
V\left(-x,-x^{\prime}\right)=V\left(x, x^{\prime}\right)
$$

in general.

Secondly, that the model is one-dimensional is not essential. For example, the model can be a two-channel scattering in three-dimensional space. In this case, the $V\left(x, x^{\prime}\right)$ is replaced by another $2 \times 2$ matrix potential $V\left(\mathbf{r}, \mathbf{r}^{\prime}\right)$, while the symmetry of the $V\left(x, x^{\prime}\right)$ becomes the condition that this $V\left(\mathbf{r}, \mathbf{r}^{\prime}\right)$ is rotationally symmetrical.

While this rotational symmetry is probably not necessary, this symmetry does play an important role. In the one-dimensional case studied in detail in Sec. V1, the symmetry of the $V\left(x, x^{\prime}\right)$, coming from $g_{2}=0$, makes it possible to use the even wave and the odd wave. Similarly, in three dimensions, the rotational symmetry of $V\left(\mathbf{r}, \mathbf{r}^{\prime}\right)$ makes it possible to use partial waves: the various partial waves do not couple so that each partial "in" wave leads to only the corresponding partial "out" wave.

Consider now the more general setting. Let the quantum memory be in a pure state $\sum_{j} a_{j}|j\rangle$, where $|j\rangle$ is a complete set of linearly independent states for the memory. The standard state $s$ is a particular linear combination of these $|j\rangle$. Let $\psi$ denote the wave function sent in from the outside to interact with the quantum memory; then the "in" field for the scattering process on the memory is

$$
\Psi^{\text {in }}=\left(\sum_{j} a_{j}^{\text {in }}|j\rangle\right) \psi^{\text {in }} .
$$

An example of $\Psi^{\text {in }}$ is given by Eq. (6.23). It should be emphasized that $\psi^{\text {in }}$ is at our disposal to accomplish whatever the purpose of this scattering is. 
It is the fundamental characteristic of the scattering from a quantum memory that not only is the "in" field $\Psi^{\text {in }}$ of the form of Eq. (7.1), but also the "out" field is of a similar form,

$$
\Psi^{\text {out }}=\left(\sum_{j} a_{j}^{\text {out }}|j\rangle\right) \psi^{\text {out }} .
$$

For the special model of Sec. VI, this important point has been discussed near the end of that section. For the present generalization, it is worked out in detail in Appendix B. As already seen in Sec. VI, Eq. (7.2) puts strong conditions on $\psi^{\text {in }}$. More precisely, a $\psi^{\text {in }}$ is defined to be admissible if, for all $\sum_{j} a_{j}^{\text {in }}|j\rangle$, the corresponding $\Psi^{\text {out }}$ is a tensor product as given by Eq. (7.2).

It should be added parenthetically that this definition of being admissible can be easily generalized by restricting the $\sum_{j} a_{j}^{\text {in }}|j\rangle$ to certain subsets. This generalization is expected to be useful in future investigations, but is not needed for this paper.

In order to perform the operations of writing, reading, and resetting a quantum memory, it is necessary to have a sufficiently large collection of admissible $\psi^{\text {in }}$. This has been verified to be the case for the model of Sec. VI, and will be assumed to be so in this section. Let

$$
\psi^{\text {in }}\left(\sum_{j} a_{j}^{\text {in }}|j\rangle \rightarrow \sum_{j} a_{j}^{\text {out }}|j\rangle\right)
$$

denote a $\psi^{\text {in }}$ with the property that, if Eq. (7.1) holds for this $\psi^{\text {in }}$, then Eq. (7.2) holds. It is assumed that, given any $a_{j}^{\text {in }}$ and $a_{j}^{\text {out }}$, there is at least one such $\psi^{\text {in }}$. It is possible that there is more than one such $\psi^{\text {in }}$. It has been seen from the model of Sec. V1 that this $\psi^{\text {in }}$ may actually involve a sequence of $\psi^{\text {in }}$ 's; see especially Eq. (6.11). However, for simplicity of notation, the expression (7.3) will be retained.

The operations of writing, reading, and resetting are now to be described in this order. For the purpose of writing after the quantum memory has been reset to the standard state $s$, it is sufficient to use any one of the $\psi^{\text {in }}\left(s \rightarrow \sum_{j} a_{j}|j\rangle\right)$, where $\sum_{j} a_{j}|j\rangle$ is the desired quantum state to be put in the memory.

Reading from a quantum memory is more complicated. Let a quantum memory be in a state $\sum_{j} a_{j}^{\text {in }}|j\rangle$; it is desired to determine the values of these $a_{j}^{\text {in }}$ by interrogating this memory, i.e., by sending a suitably chosen sequence of admissible $\psi^{\text {in }}$ 's,

$$
\psi^{\operatorname{in}(1)}, \psi^{\operatorname{in}(2)}, \psi^{\operatorname{in}(3)}, \cdots, \psi^{\operatorname{in}(N)},
$$

and scattering them successively by this quantum memory. More precisely, consider 
the successive scattering processes

$$
\begin{aligned}
\sum_{j} a_{j}^{\text {in }}|j\rangle \psi^{\text {in(1) }} \rightarrow \sum_{j} a_{j}^{\text {out(1) }}|j\rangle \psi^{\text {out }(1)} \rightarrow a_{j}^{\text {out }(1)}=a_{j}^{\text {in }(2)} \\
\rightarrow \sum_{j} a_{j}^{\text {in }(2)}|j\rangle \psi^{\text {in(2) }} \rightarrow \sum_{j} a_{j}^{\text {out }(2)}|j\rangle \psi^{\text {out }(2)} \rightarrow a_{j}^{\text {out }(2)}=a_{j}^{\text {in }(3)} \\
\rightarrow \sum_{j} a_{j}^{\text {in }(3)}|j\rangle \psi^{\text {in(3) }} \rightarrow \sum_{j} a_{j}^{\text {out }(3)}|j\rangle \psi^{\text {out }(3)} \rightarrow a_{j}^{\text {out }(3)}=a_{j}^{\text {in }(4)} \\
\rightarrow \cdots \\
\rightarrow \sum_{j} a_{j}^{\text {in }(N)}|j\rangle \psi^{\text {in }(N)} \rightarrow \sum_{j} a_{j}^{\text {out }(N)}|j\rangle \psi^{\text {out }(N)} .
\end{aligned}
$$

Corresponding to the list (7.4), there is a list of $\psi^{\text {out }}$ 's,

$$
\psi^{\text {out }(1)}, \psi^{\text {out }(2)}, \psi^{\text {out }(3)}, \cdots, \psi^{\text {out }(N)} \text {. }
$$

From the quantities given in (7.4) and (7.6) together with their interference, the values of $a_{j}^{\text {in }}$ are obtained. This has been demonstrated explicitly in Sec. VI for the model there, and it is also shown there that a further interference with the standard state $s$ may be needed to determine the overall phase. The importance of interference cannot be over-emphasized.

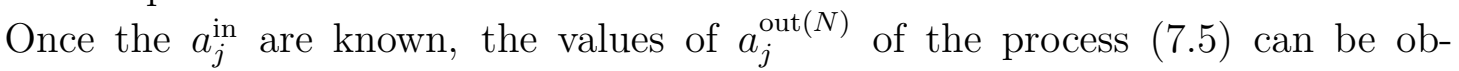
tained. An additional scattering using any one of the admissible $\psi^{\text {in }}\left(\sum_{j} a_{j}^{\text {out }(N)}|j\rangle \rightarrow\right.$ $\left.\sum_{j} a_{j}^{\text {in }}|j\rangle\right)$ returns the quantum memory to its initial state.

With the above process of reading a quantum memory, resetting is now straightforward. Resetting a quantum memory in the initial state $\sum_{j} a_{j}^{\text {in }}|j\rangle$ to the standard state $s$ consists of the following two steps.

(i) Read the memory to determine $a_{j}^{\text {in }}$. Note that, after the process of reading is performed, the memory is in the original initial state $\sum_{j} a_{j}^{\text {in }}|j\rangle$.

(ii) Apply an additional scattering using any one of the admissible $\psi^{\text {in }}\left(\sum_{j} a_{j}^{\text {in }}|j\rangle \rightarrow\right.$ $s)$.

This completes the description of the quantum memory together with writing, reading, and resetting, all performed through scattering from the memory.

It may be worthwhile to emphasize that the concept of the quantum memory introduced and described here is quite general. In particular, the scattering process

$$
\Psi^{\text {in }}=\left(\sum_{j} a_{j}^{\text {in }}|j\rangle\right) \psi^{\text {in }} \rightarrow \Psi^{\text {out }}=\left(\sum_{j} a_{j}^{\text {out }}|j\rangle\right) \psi^{\text {out }}
$$

does not have many restrictions, and may or may not be linear. Also, the linearly independent states $|j\rangle$ are allowed to depend on time, and may or may not be the eigenstates of an operator. 


\section{COMPARISON WITH AN EARLIER MODEL}

The idea of quantum computing was first discussed by Benioff in 1980 [12]. In this pioneering paper, spatial dependence was retained, although not quite in the form of the Schrödinger equation. Since then, quantum computing and quantum information have become popular subjects with a vast literature [13]. However, in the majority of the theoretical papers on quantum computing, spatial dependence is omitted entirely. Therefore the usual model for quantum memory consists of a spin system or its generalization, and the operations on the quantum memory consist of applying unitary matrices. This prevailing model for the quantum memory has led to a number of important results.

In the present paper, as a first application of the Fermi pseudo-potential in one dimension, an alternative model for the quantum memory is proposed. This model differs from the previous one mainly in the re-introduction of the spatial variables, much in the spirit of the original work of Benioff [12]. From the point of view of physics, the spatial variables are clearly present, whether one wants them or not. Instead of saying that a unitary matrix is applied mathematically to the content of the quantum memory, here the content of the quantum memory is altered in a controlled way by applying suitably chosen scatterings to the memory.

This is much more than a change of language. While the previous model has the advantage of simplicity, which is important because quantum computing is a difficult subject, the present model with the spatial variable or variables may be considered to be desirable from the following two points of view. First, it offers a closer description of the experimental situation. Since a quantum memory is necessarily small in size, for practical reasons scattering is the simplest means of modifying the content of a quantum memory. Secondly, the presence of the spatial dimensions allows more possibilities of analyzing the quantum memory. It is also worth mentioning that the theory of scattering has been developed over many decades and is well understood, in the context of both quantum mechanics and quantum field theory. It is often advantageous to be able to make use of existing knowledge to study a new subject.

In both the previous model and the present model, the content of a quantum memory is given as a pure state. This content is altered by applying a unitary transformation, directly in the previous model and indirectly through scattering in the present model. The incident, scattered, and total wave functions have no analog in the previous model. In general, the phase shift [2] of scattering is determined from the total wave function, and the analysis of the explicit model in Sec. VI is actually an especially simple application of the usual phase-shift analysis, including the prominent role played by interference. The point is that, while in the definition of an admissible $\psi^{\text {in }}$ in Sec. VII both $\Psi^{\text {in }}\left[\right.$ Eq. (7.1)] and $\Psi^{\text {out }}[$ Eq. (7.2)] are unentangled so far as the memory and the interrogating wave are concerned, this is not true of the total wave function, which is for example

$$
\psi(x)=\left[\begin{array}{l}
\psi_{1}(x) \\
\psi_{2}(x)
\end{array}\right]
$$

for the model of Sec. V1.

There are many interesting open questions for the present model. The analysis of 
these questions is beyond the scope of the present paper. Nevertheless, here are two examples of such open questions.

(a) In Sec. VII, it is explicitly assumed that there is a sufficiently large class of admissible $\psi^{\text {in }}$ of the form (7.3). In the model of Sec. VI, such a large class indeed exists in the form of even waves and odd waves. On the other hand, when $g_{2} \neq 0$, no such large class exists. What is needed is a more general discussion as to the conditions under which such a sufficiently large class of admissible $\psi^{\text {in }}$ is actually available.

Even though examples where such a large class is available are known both in one dimension and in three dimensions, the three-dimensional case seems rather difficult to achieve experimentally. If this observation is true in general, then there may well be significant advantages to connecting the various components of a quantum computer, including quantum memories, by single-mode optical fibers. In particular, sending signals through space rather than fibers may lead to unexpected problems.

(b) Another especially challenging and interesting question for the present model of quantum memory concerns the issue of the so-called no-cloning theorem. This has been derived in the context of the previous model, but such derivations do not seem to be applicable directly to the present model. This is again related to the fact that here there is not only an $S$-matrix but also the incident, scattered, and total wave functions.

Preliminary analysis indicates that whether the no-cloning theorem holds for the present model of quantum memory may depend on subtle aspects of the Schrödinger equation. If this is indeed the case, then the no-cloning theorem may need to be stated properly and precisely before it can be derived within the present model of quantum memory.

\section{DISCUSSIONS}

The present investigation began as an attempt to understand the $\delta^{\prime}(x)$ potential in the context of the one-dimensional Schrödinger equation. When simple attempts failed, the powerful method of the resolvent equation was used. The surprise is that, not only can the resolvent equation be solved in general in terms of rational functions, but also the solution yields, in addition to the well-known $\delta$-function potential, not one but two linearly independent Fermi pseudo-potentials in one dimension. One of the pseudo-potentials is odd under space reflection and is the proper interpretation of the $\delta^{\prime}(x)$ potential. The other one is originally unexpected and is even under space reflection.

It is likely that there are many applications of these pseudo-potentials to onedimensional problems. A possible use in statistical mechanics connected with the Bethe ansatz [8] has already been mentioned in Sec. V1. In this paper, only the simplest application is discussed. This has nothing to do with the proper interpretation of the $\delta^{\prime}(x)$ potential, but depends critically on the unexpected, even pseudo-potential. By combining this even pseudo-potential with the $\delta$-function potential, an elegant special case is found for the scattering in two coupled channels. Even though the two channels cannot be decoupled, it is easy to write down the complete solution from the known one-channel case. 
In spite of the mathematical simplicity of this application of the Fermi pseudopotential in one dimension, this example gives a model for the quantum memory (sometimes called the quantum register). While this model is completely explicit, its more important function is to point out a way to gain a general picture concerning the quantum memory.

More generally, the time-independent Schrödinger equation for $n$ coupled channels with interaction at only the one point $x=0$ is

$$
-\frac{d^{2} \psi(x)}{d x^{2}}+\int_{-\infty}^{\infty} d x^{\prime} V\left(x, x^{\prime}\right) \psi\left(x^{\prime}\right)=k^{2} \psi(x)
$$

with

$$
\psi(x)=\left[\begin{array}{c}
\psi_{1}(x) \\
\psi_{2}(x) \\
\vdots \\
\psi_{n}(x)
\end{array}\right]
$$

and

$$
V\left(x, x^{\prime}\right)=C_{1} \delta(x) \delta\left(x^{\prime}\right)+C_{2}\left[\delta_{p}^{\prime}(x) \delta\left(x^{\prime}\right)+\delta(x) \delta_{p}^{\prime}\left(x^{\prime}\right)\right]+C_{3} \delta_{p}^{\prime}(x) \delta_{p}^{\prime}\left(x^{\prime}\right) .
$$

Here $C_{1}, C_{2}$ and $C_{3}$ are three numerical hermitian $n \times n$ matrices, while $\delta_{p}^{\prime}(x)$ is similar to $\delta^{\prime}(x)$ and is defined in Sec. IV. For a given $k$ and a given incident wave $\psi_{0}(x)$ with $n$ components, the solution of Eq. (9.1) takes the form, for $j=1,2, \ldots$, $n$,

$$
\psi_{j}(x)=\psi_{0 j}(x)+ \begin{cases}F_{j+} e^{i k x}, & \text { for } x>0 \\ F_{j-} e^{-i k x}, & \text { for } x<0,\end{cases}
$$

analogous to Eq. (2.7), where the $F$ 's are $2 n$ coefficients that depend on $\psi_{0 j}$ and $k$. The substitution of Eq. (9.4) into Eq. (9.1) shows that these $2 n F^{\prime}$ 's satisfy $2 n$ linear equations. Indeed, it is the power of the Fermi pseudo-potential that Schrödinger equations reduce to linear algebraic equations. It will be interesting to study the structure of these algebraic equations. Even more generally, the pseudo-potential (9.3) at $x=0$ may be replaced by a linear superposition of a finite number of such pseudo-potentials at $x=x_{1}, x_{2}, \ldots$. The number of coefficients in the solution increases but remains finite, leading to more simultaneous algebraic equations that are still linear. The Green's functions can be treated in a very similar manner.

Needless to say, the range of integration in Eq. (9.1) for $x^{\prime}$ can be replaced by a semi-infinite or finite interval, and the $V\left(x, x^{\prime}\right)$ may contain additional terms such as those from step potentials. A more interesting problem is to apply the Fermi pseudo-potentials to first-order differential equations.

In summary, the theory of the Fermi pseudo-potential in one dimension has been worked out here together with the simplest non-trivial application to a problem of current interest. 


\section{Acknowledgments}

For helpful discussions, we are indebted to Maurice Jacob, Harold Levine, André Martin, John Myers, and Raymond Stora. One of us (TTW) thanks the Theoretical Physics Division of CERN for their kind hospitality. Some of the thinking in this paper has been discussed in an unpublished Harvard preprint by John Myers and TTW.

\section{APPENDIX A}

A possible way to solve Eq. (3.13) for $F(\kappa)$ is as follows. Because of the exponential function in the last term, let

$$
x=e^{F(\kappa)}
$$

and

$$
y=\ln \kappa
$$

[This $x$ of Eq. (A1) of course has nothing to do with the space variable in the Schrödinger equation (1.1), for example.] In view of Eq. (3.3), Eq. (3.13) is translationally invariant in $y$. It is therefore desirable to use $x$ as the independent variable

and $y$ as the dependent variable, leading to a first-order ordinary differential equation for

$$
z=\frac{d y}{d x}
$$

This first-order equation is

$$
-\frac{2}{x^{2} z^{3}}\left[x \frac{d z}{d x}+z\right]=\frac{1}{x^{2} z^{2}}-1+\left(c_{3}^{2}+c_{2} c_{4}\right) x^{2} .
$$

Let

$$
u=\frac{1}{x^{3} z^{2}}
$$

Then, after some algebra,

$$
\frac{d u}{d x}=-\frac{1}{x^{2}}+\left(c_{3}^{2}+c_{2} c_{4}\right)
$$

Integration yields

$$
u=\frac{1}{x}+2 c_{1}+\left(c_{3}^{2}+c_{2} c_{4}\right) x,
$$

where $c_{1}$ is the fourth arbitrary constant of integration. The expression for $z$ follows from Eqs. (A5) and (A7):

$$
z= \pm x^{-1}\left[1+2 c_{1} x+\left(c_{3}^{2}+c_{2} c_{4}\right) x^{2}\right]^{-1 / 2},
$$


where the \pm sign comes from taking the square root of $z^{2}$. It is, of course, related to the fact that every term in Eq. (A4) is even in $z$. In the following, there are many \pm and $\mp$ signs; it is to be understood that these signs are used as a shorthand for two equations, one with the upper sign everywhere and a second one with the lower sign everywhere.

From Eqs. (A3) and (A8), $y$ is given by

$$
y=\mp \int \frac{d x^{\prime}}{\sqrt{\left(c_{3}^{2}+c_{2} c_{4}\right)+2 c_{1} x^{\prime}+x^{\prime 2}}},
$$

where $x^{\prime}=1 / x$. It is fortunate that this integral is elementary. For definiteness, consider the case

$$
c_{3}^{2}+c_{2} c_{4}-c_{1}^{2}>0
$$

In this case, the explicit integration of the right-hand side of Eq. (A9) gives

$$
y=\mp\left[\sinh ^{-1} \frac{1+c_{1} x}{\sqrt{c_{3}^{2}+c_{2} c_{4}-c_{1}^{2} x}}+\text { const. }\right] .
$$

The rest of the calculation is straightforward although lengthy, and the results are

$$
\begin{aligned}
& f_{1}(\kappa)=\frac{-\sqrt{c_{3}^{2}+c_{2} c_{4}-c_{1}^{2}}\left[c_{0} \kappa+\left(c_{0} \kappa\right)^{-1}\right] \mp 2 c_{3}}{\sqrt{c_{3}^{2}+c_{2} c_{4}-c_{1}^{2}}\left[c_{0} \kappa-\left(c_{0} \kappa\right)^{-1}\right] \pm 2 c_{1}}, \\
& f_{2}(\kappa)=1 \mp \frac{2 c_{2}}{\sqrt{c_{3}^{2}+c_{2} c_{4}-c_{1}^{2}}\left[c_{0} \kappa-\left(c_{0} \kappa\right)^{-1}\right] \pm 2 c_{1}}, \\
& f_{3}(\kappa)=\frac{-\sqrt{c_{3}^{2}+c_{2} c_{4}-c_{1}^{2}}\left[c_{0} \kappa+\left(c_{0} \kappa\right)^{-1}\right] \pm 2 c_{3}}{\sqrt{c_{3}^{2}+c_{2} c_{4}-c_{1}^{2}}\left[c_{0} \kappa-\left(c_{0} \kappa\right)^{-1}\right] \pm 2 c_{1}}, \\
& f_{4}(\kappa)=1 \mp \frac{2 c_{4}}{\sqrt{c_{3}^{2}+c_{2} c_{4}-c_{1}^{2}}\left[c_{0} \kappa-\left(c_{0} \kappa\right)^{-1}\right] \pm 2 c_{1}} .
\end{aligned}
$$

Since $c_{1}, c_{2}, c_{3}$ and $c_{4}$ are arbitrary constants of integration, their signs can be changed simultaneously. This change then removes all the \pm and $\mp$ signs, and Eqs. (A12)(A15) reduce to Eqs. (3.14).

The other case where $c_{3}^{2}+c_{2} c_{4}-c_{1}^{2}<0$ can be treated in an entirely similar manner, leading to Eq. (3.16).

\section{APPENDIX B}

In this Appendix B, the reasoning is given that leads to Eq. (7.2).

Just as the $\Psi^{\text {in }}$ of Eq. (7.1) contains information about the initial state of the quantum memory and the behavior of the incoming wave $\psi^{\text {in }}$ before scattering, the $\Psi^{\text {out }}$ contains information about the final state of the quantum memory and the behavior of the outgoing wave after scattering. Later the outgoing wave moves away 
from the memory and the information about this outgoing wave is no longer available. This means that the final state of the memory is given by $\Psi^{\text {out }}$ averaged over this outgoing wave. This average can be written schematically as

$$
M=\int \Psi^{\text {out }} \Psi^{\text {out } \dagger}
$$

This $M$ is the density matrix for the quantum memory. Here the $\int$ indicates integration and summation over all degrees of freedom associated with the outgoing wave or particle, but not those of the quantum memory. The corresponding differential symbol is omitted: It is $d^{3} \mathbf{r}$ if the wave is described by the three-dimensional Schrödinger equation; it is $d^{3} \mathbf{r}$ together with a summation over the spin in the case of the Dirac equation; and it is a functional differential such as $\mathcal{D} A_{\mu}$ in the context of quantum field theory.

In order for the quantum memory to function, the final state must be a pure state $\sum_{j} a_{j}^{\text {out }}|j\rangle$, just like the initial state. Therefore, the above $M$ must also be given by

$$
M=\sum_{j} a_{j}^{\text {out }}|j\rangle \sum_{i} a_{i}^{\text {out* }}\langle i|
$$

Equating the two formulas for $M$ gives

$$
\sum_{j} a_{j}^{\text {out }}|j\rangle \sum_{i} a_{i}^{\text {out* }}\langle i|=\int \Psi^{\text {out }} \Psi^{\text {out } \dagger} .
$$

It remains to derive Eq. (7.2) from Eq. (B3).

Since the $|j\rangle$ form a complete set, $\Psi^{\text {out }}$ can always be written in the form

$$
\Psi^{\text {out }}=\sum_{j}|j\rangle \psi_{j}^{\text {out }}
$$

The substitution of Eq. (B4) into Eq. (B3) gives

$$
a_{j}^{\text {out }} a_{i}^{\text {out* }}=\int \psi_{j}^{\text {out }} \psi_{i}^{\text {out } \dagger}
$$

for all $i$ and $j$. For $i \neq j$, define the integral

$$
I_{j i}=\int\left(a_{i}^{\text {out }} \psi_{j}^{\text {out }}-a_{j}^{\text {out }} \psi_{i}^{\text {out }}\right)\left(a_{i}^{\text {out } *} \psi_{j}^{\text {out } \dagger}-a_{j}^{\text {out } *} \psi_{i}^{\text {out } \dagger}\right) .
$$

This $I_{j i}$ is non-negative, and is zero only if

$$
a_{i}^{\text {out }} \psi_{j}^{\text {out }}-a_{j}^{\text {out }} \psi_{i}^{\text {out }}=0 .
$$

But the substitution of Eq. (B5) into Eq. (B6) gives immediately that

$$
I_{j i}=0 \text {. }
$$

Thus, Eq. (B7) holds for all $i$ and $j$.

The desired Eq. (7.2) is just Eq. (B4) with Eq. (B7). 
[1] N. N. Khuri and T. T. Wu, Phys. Rev. D 56, 6779 and 6785 (1997).

[2] J. M. Blatt and V. F. Weisskopf, Theoretical Nuclear Physics (John Wiley and Sons, New York, 1952).

[3] K. Huang and C. N. Yang, Phys. Rev. 105, 767 (1957).

[4] T. D. Lee and C. N. Yang, Phys. Rev. 105, 1119 (1957).

[5] T. D. Lee, K. Huang, and C. N. Yang, Phys. Rev. 106, 1135 (1957).

[6] T. T. Wu, Phys. Rev. 115, 1390 (1959).

[7] G. Flamand, in 1965 Cargèse Lectures in Theoretical Physics, edited by F. Lurçat (Gordon and Breach Science Publishers, New York, 1967).

[8] H. A. Bethe, Z. Phys. 71, 205 (1931).

[9] C. N. Yang and C. P. Yang, J. Math. Phys. 10, 1115 (1969).

[10] G. E. Moore, Electronics 38, 114 (1965).

[11] R. Pike and E. P. Sabatier (Eds.), Scattering-Scattering and Inverse Scattering in Pure and Applied Science (Academic Press, London, 2002).

[12] P. Benioff, J. Statistical Phys. 22, 563 (1980).

[13] A small selection of papers are: P. Benioff, J. Math. Phys. 22, 495 (1981); D. Z. Albert, Phys. Lett. A 98, 249 (1983); R. P. Feynman, Optics News 11 (February 1985); D. Deutsch, Proc. R. Soc. London, Part A 400, 97 (1985); P. W. Shor, Phys. Rev. A 52, 2493 (1995); S. Parker and M. B. Plenio, Phys. Rev. Lett. 85, 3049 (2000); and X. Wang, A. Sorenson, and K. Mølmer, Phys. Rev. Lett. 86, 3907 (2001). 